\title{
Treatment of Overlap Syndromes in Autoimmune Liver Disease: A Systematic Review and Meta-Analysis
}

\author{
Benjamin L. Freedman ${ }^{1}$, Christopher J. Danford ${ }^{2}\left(\mathbb{D}\right.$, Vilas Patwardhan ${ }^{3}\left(\mathbb{C}\right.$ and Alan Bonder ${ }^{3, *} * \mathbb{C}$ \\ 1 Department of Medicine, Beth Israel Deaconess Medical Center, 330 Brookline Ave., Boston, MA 02215, USA; \\ bfreedm1@bidmc.harvard.edu \\ 2 Division of Gastroenterology and Hepatology, Beth Israel Deaconess Medical Center, 330 Brookline Ave., \\ Dana 603, Boston, MA 02215, USA; cdanford@bidmc.harvard.edu \\ 3 Liver Center, Autoimmune and Cholestatic Liver Disease Program, Department of Medicine, Beth Israel \\ Deaconess Medical Center, Harvard Medical School, 110 Francis St. Suite 8E, Boston, MA 02215, USA; \\ vpatward@bidmc.harvard.edu \\ * Correspondence: abonder@bidmc.harvard.edu; Tel.: +1-617-632-1070
}

Received: 13 April 2020; Accepted: 7 May 2020; Published: 13 May 2020

\begin{abstract}
The treatment of overlap syndromes is guided by small observational studies whose data have never been synthesized in a rigorous, quantitative manner. We conducted a systematic review and meta-analysis to evaluate the efficacy of available treatments for these rare and morbid conditions. We searched the literature for studies comparing $\geq 2$ therapies for autoimmune hepatitis (AIH)-primary biliary cholangitis (PBC), AIH-primary sclerosing cholangitis (PSC), PBC-PSC, AIH-PBC-PSC, autoimmune cholangitis (AIC), or autoimmune sclerosing cholangitis (ASC) with respect to various clinical outcomes, including biochemical improvement and transplant-free survival. A total of 28 studies met the inclusion criteria for AIH-PBC, AIH-PSC, AIC, and ASC. AIH-PBC patients tended to experience more biochemical improvement with ursodeoxycholic acid (UDCA) + [corticosteroids and/or antimetabolites], i.e., "combination therapy", than with corticosteroids \pm azathioprine ( $\mathrm{RR}=4.00,95 \% \mathrm{CI} 0.93-17.18)$. AIH-PBC patients had higher transplant-free survival with combination therapy than with UDCA, but only when studies with follow-up periods $\leq 90$ months were excluded ( $R R=6.50,95 \%$ CI 1.47-28.83). Combination therapy may therefore be superior to both UDCA and corticosteroids \pm azathioprine for the treatment of AIH-PBC, but additional studies are needed to show this definitively and to elucidate optimal treatments for other overlap syndromes.
\end{abstract}

Keywords: overlap syndrome; autoimmune liver disease; immunosuppression; corticosteroid; ursodeoxycholic acid

\section{Introduction}

The term "overlap syndromes" is used to describe a family of rare, morbid conditions characterized by biochemical, immunologic, histologic, or cholangiographic features of more than one of the well-recognized autoimmune liver diseases: Autoimmune hepatitis (AIH), primary biliary cholangitis (PBC), and primary sclerosing cholangitis (PSC) [1]. These overlap syndromes include AIH-PBC, AIH-PSC, PBC-PSC, AIH-PBC-PSC, autoimmune cholangitis (AIC), and autoimmune sclerosing cholangitis (ASC). While their initial presentations differ, any of them may ultimately progress to cirrhosis and its sequelae.

AIH-PBC is the most studied of these disorders, occurring in 1-3\% of patients with PBC [2] and $7 \%$ of patients with $\mathrm{AIH}$ [3]. Based upon histologic, biochemical, and/or immunologic features of each parent disorder, the Paris criteria are the most widely accepted means of diagnosing AIH-PBC [4]. 
Most investigators to date have chosen a first-line treatment regimen including both ursodeoxycholic acid (UDCA) and corticosteroids, with or without azathioprine (AZA), often with encouraging results [2,4-12]. However, in some studies patients appeared to benefit from UDCA alone $[7,10,13]$, or from just corticosteroids with or without AZA [3,14]. UDCA has been augmented with tacrolimus [10], mycophenolate mofetil (MMF) $[15,16]$, or cyclosporine $[8,10,17]$ as second line therapies, with some success, in patients intolerant of or unresponsive to corticosteroids and/or AZA. At 10 years, progression to cirrhosis among patients with AIH-PBC is approximately $44-48 \%[18,19]$, and transplant-free survival is $52-92 \%$ [19-21].

AIC, also known as AIH-cholestatic overlap syndrome, is typically defined by the coexistence of AIH and a cholestatic syndrome that does not meet criteria for either classic PBC or PSC: alkaline phosphatase (AP) and $\gamma$-glutamyltransferase (GGT) are elevated and liver biopsy shows bile duct injury (in various histologic patterns), but anti-mitochondrial antibodies (AMA) are absent and cholangiography is normal [1]. Czaja et al. therefore postulate that, in some cases of AIC, AMA-negative PBC or small-duct PSC may be the true clinical entities overlapping with AIH [1]. AIC has been identified in $1 \%$ and $11 \%$ of patients with PBC [22] and AIH [3], respectively. The same first line treatment options exist for AIC as for AIH-PBC (UDCA [23], corticosteroids \pm AZA [3,22,24], or a combination of both $[23,25])$, although AIC has shown a poorer response to treatment across the board. Limited prognostic data suggest poor clinical outcomes as in one study, two out of six patients with AIC experienced either liver-related death or transplant after a median of three months [3] and in another, one of 20 patients with AIC died from liver failure seven months into follow-up [24].

The diagnosis of AIH-PSC requires the coexistence in adult patients of AIH (defined in various studies by the original [26], revised [27], or simplified [28] International Autoimmune Hepatitis Group [IAIHG] criteria) and cholangiographic or histologic features of PSC [29]. AIH-PSC has been identified in $1.4-17 \%$ of adults with PSC [29]. Among adults with AIH, the prevalence of AIH-PSC depends upon the presence $(44 \%)$ or absence $(8 \%)$ of comorbid inflammatory bowel disease [30]. This syndrome is more common in children, where it has been termed ASC: Up to 50\% of children with AIH have cholangiographic features of PSC [31]. In AIH-PSC, as in AIH-PBC, a treatment regimen combining UDCA and corticosteroids (with or without AZA) has been used most frequently, although with a variable clinical response [32-38]. UDCA alone [33,38-40], or corticosteroids with or without AZA $[3,33,35,40-42]$, have also been used, albeit less often. Similarly, a combination of UDCA and corticosteroids (with or without AZA) is the most common treatment regimen for ASC [33,36,43,44], while UDCA monotherapy has been used in some cases $[33,43,44]$. Limited experience using MMF as second-line therapy for patients unresponsive to, or intolerant of, corticosteroids or AZA suggests a greater efficacy for AIH-PSC [16] than for ASC [45]. At 10 years, overall survival is higher for ASC $(89 \%)$ [36] than for AIH-PSC ( 67\%) [46], while transplant-free survival is comparable between the two $(65 \%$ vs. $63 \%)[36,40]$.

PBC-PSC [47-53] and AIH-PBC-PSC [50,54] have also been reported in the literature, although the prevalence of these overlap syndromes is unknown. While there are as yet no standardized diagnostic criteria for these disorders, PBC-PSC is generally characterized by a cholestatic laboratory profile, AMA positivity and/or histologic characteristics of $\mathrm{PBC}$, and cholangiographic and/or histologic features of PSC. AIH-PBC-PSC contains the features above in addition to biochemical, immunologic, and/or histologic evidence of AIH. PBC-PSC has been treated primarily with UDCA monotherapy, although prednisolone/AZA or adalimumab have been used concurrently with UDCA in patients with comorbid rheumatoid arthritis or psoriatic arthritis, respectively $[48,49]$. While prognostic data on PBC-PSC are sparse, one patient who presented with cirrhosis died from liver failure five years later [50], another patient developed cirrhosis within four years of symptom onset [47], and two patients experienced cholangiographic progression despite biochemical improvement on UDCA monotherapy [48,51]. AIH-PBC-PSC has been treated with either prednisolone and AZA [50], UDCA combined with budesonide and AZA [54], or UDCA combined with prednisone and AZA [55]. The prognosis of this rare disorder is not yet clear. 
The rarity of overlap syndromes in autoimmune liver disease diminishes the ease but not the importance of rigorously studying their optimal treatment regimens. Indeed, in many respects overlap syndromes carry worse prognoses than their parent diseases. For example, one cohort study showed that death or liver transplantation occurred in $38 \%$ of patients with AIH-PBC but only $19 \%$ of patients with PBC during the six-year mean follow-up period $(p<0.05)$ [20]. In a different study, during $\sim 26$ months of follow-up, 33\% of patients with AIH-PSC experienced liver-related death or transplant, compared to only $8 \%$ of those with AIH $(p=0.05)$ [3]. To our knowledge, the primary literature on the treatment of overlap syndromes is devoid of randomized trials and consists entirely of observational cohort studies, case series, and case reports. Zhang et al. published two meta-analyses on the treatment of AIH-PBC [12,56], but both contain serious methodological flaws. Both, for example, mislabel non-randomized cohort studies $[4,6,8,10,25,57,58]$ as randomized controlled trials, and double-count study participants by including a pair of studies with overlapping patient cohorts $[4,6]$. The more recent of the two meta-analyses [12] purports to examine the effects of UDCA/budesonide combination therapy, but includes several studies in which budesonide is never mentioned $[8,10,25]$.

The treatment recommendations of the American Association for the Study of Liver Diseases (AASLD) and the European Association for the Study of the Liver (EASL) for overlap syndromes in autoimmune liver disease are relatively sparse, reflecting a thin base of primary evidence. For example, the EASL 2017 PBC guidelines state that "Patients with PBC and typical features of AIH may (emphasis added) benefit from immunosuppressive treatment in addition to UDCA" and recommend that immunosuppression be given, or considered, in patients with severe or moderate interface hepatitis, respectively [59]. The AASLD 2018 PBC guidelines concede that "the clinical benefit and harm of adding immunosuppressive medications to PBC patients with AIH features require further study", and recommend tailoring pharmacotherapy to the predominant histologic pattern of injury [60]. For AIH-PSC and ASC, the AASLD 2010 PSC guidelines recommend "corticosteroids and other immunosuppressive agents", while acknowledging that the impact of these medications remains unclear [29]. The EASL 2015 AIH guidelines further recommend that the addition of UDCA to immunosuppression can be considered, although "It is difficult to draw any firm conclusions because of the small number of patients, the usually retrospective nature of the studies and the heterogeneity of the regimens" [31]. No guidelines exist for the optimal treatment of AIC, PBC-PSC, or AIH-PBC-PSC.

Given the ambiguity of current guidelines, the limited power of individual studies, and dubious quality of existing meta-analyses on the pharmacotherapy of overlap syndromes, an updated synthesis of the primary literature is warranted to guide optimal treatment strategies. We therefore conducted a broad systematic review and meta-analysis of all medications used to treat AIH-PBC, AIH-PSC, PBC-PSC, AIH-PBC-PSC, AIC, or ASC, comparing the efficacy of different treatment regimens for each overlap syndrome as measured by symptomatic, biochemical, histologic, and transplant-free survival outcomes.

\section{Materials and Methods}

This systematic review and meta-analysis are reported in accordance with the Preferred Reporting Items for Systematic Reviews and Meta-Analyses (PRISMA) statement [61].

\subsection{Selection Criteria}

For our systematic review, we included only those studies published as full-text articles in peer-reviewed journals, either in English or with an accessible English translation. Randomized trials and observational cohort studies were accepted, while case reports, case series, and review articles were not. Studies were required to compare two or more pharmacotherapies in human subjects for the first-line treatment of at least one of the following overlap syndromes: AIH-PBC, AIH-PSC, PBC-PSC, AIH-PBC-PSC, AIC, or ASC. Eligible studies also had to report clinical outcomes using at least one of the following parameters: Symptomatic improvement, biochemical improvement, improvement in histologic activity, non-progression of liver fibrosis, or transplant-free survival. There were no 
restrictions on the age or ethnicity of study participants, the diagnostic criteria used to define overlap syndromes, or the definitions of clinical outcomes.

\subsection{Data Sources and Search Strategy}

Our detailed search strategies were designed with input from an experienced medical librarian and can be found in Table A1. The medical databases Cochrane Library, EMBASE, PubMed, and Web of Science were queried from inception through 30 September, 2019 using the following search terms (and synonyms thereof) in various combinations: "autoimmune liver disease", "overlap syndrome", "primary biliary cholangitis", "primary sclerosing cholangitis", "autoimmune hepatitis", "autoimmune cholangitis", and "autoimmune sclerosing cholangitis". The titles, abstracts, and/or full texts of the resulting studies were screened by one reviewer (B.F.) to determine their eligibility for the systematic review.

\subsection{Data Extraction and Quality Assessment}

The same reviewer (B.F.) extracted the following data from all included studies: Authorship; year of publication; study design; overlap syndrome(s) examined; diagnostic criteria used; duration of follow-up; treatments administered; number, age, and gender distribution of patients in each treatment group; which clinical outcome(s) were reported (and how they were defined); and the proportion of patients in each treatment group who experienced these outcomes. Whenever feasible, patients who crossed between different treatment regimens during follow-up were censored from the analysis. The quality of individual studies was assessed using the Newcastle-Ottawa Scale [62], which awards a maximum of 9 points (indicating maximal study quality), subdivided by patient selection ( $0-4$ stars), comparability of patient cohorts ( $0-2$ stars), and clinical outcomes and follow-up ( $0-3$ stars). For the latter category of the Newcastle-Ottawa Scale, we considered 2 months, 6 months, and 5 years to be sufficient durations of follow-up for biochemical, histologic, and transplant-free survival outcomes to occur, respectively. Furthermore, we felt that significant attrition bias was unlikely as long as $\leq 10 \%$ of patients were lost to follow-up in a given study.

\subsection{Statistical Analysis}

Data from individual studies were meta-analyzed using a random effects model, and heterogeneity between studies was quantified with the $\mathrm{I}^{2}$ statistic (where $\mathrm{I}^{2}>50 \%$ was considered significant heterogeneity). Separate meta-analyses were performed for each distinct combination of overlap syndrome, treatment comparison, and clinical outcome. Funnel plots were constructed to assess the likelihood of publication bias in all meta-analyses comprising $\geq 10$ studies. All statistical tests were 2-tailed, with a significance threshold of $p<0.05$. All statistical analyses were performed using Review Manager 5.3 (The Cochrane Collaboration, Copenhagen, Denmark).

\subsection{Quality of Evidence}

We used the Grading of Recommendations Assessment, Development, and Evaluation (GRADE) system to assess the quality of evidence derived from each of our meta-analyses [63]. According to the GRADE system, evidence is rated as either high, moderate, low, or very low quality depending on a number of factors. Meta-analyses of randomized trials are considered high quality by default, but may be downgraded by one or more levels if their constituent trials exhibit poor study design, excessive heterogeneity of results, limited generalizability, low precision, or publication bias. Observational studies are considered by default to be of low quality, and may be downgraded further for the reasons above or upgraded if specific features are present, such as a large magnitude of effect or a dose-response gradient.

\section{Results}

Our systematic literature search yielded 5483 unique publications, of which we included 28 in our 
systematic review and 20 in our meta-analyses (Figure A1). All publications meeting inclusion criteria for the systematic review were observational cohort studies. The systematic review comprised 20 studies pertaining to AIH-PBC [6-8,10,11,13,25,57,64-75], two pertaining to AIH-PSC [35,41], two pertaining to AIC [23,24], three pertaining to ASC [33,43,44], and one pertaining to both AIH-PBC and AIC [22]. Of the 21 studies that included patients with AIH-PBC, 17 compared UDCA monotherapy to UDCA + [corticosteroids and/or antimetabolites (AZA or MMF)], i.e., "combination therapy" [6-8,10,11,25, 57,65-71,73-75]. Five out of these 17 studies also contained a third group of patients treated with corticosteroids $\pm \mathrm{AZA}$, thereby allowing additional treatment comparisons $[8,68,71,73,74]$. Of the four remaining studies of AIH-PBC, one compared UDCA to placebo [13], two compared UDCA to corticosteroids \pm AZA [22,64], and one compared corticosteroids + AZA to combination therapy [72]. Both studies of AIH-PSC compared corticosteroids \pm AZA to combination therapy [35,41]. Of the three studies pertaining to AIC, two compared UDCA to corticosteroids \pm AZA [22,24], while the third compared combination therapy to a complex personalized regimen consisting of UDCA, prednisolone, AZA, MMF, budesonide, rifampicin, and several other agents [23]. All studies of ASC compared UDCA to combination therapy $[33,43,44]$. Among the 21 AIH-PBC studies in our systematic review, four were ineligible for meta-analysis: One because it was the only study comparing UDCA to placebo [13], one because of an ambiguous overlap between treatment groups [22], and two because no clinical outcomes occurred during follow-up [69,72]. Neither study of AIH-PSC could be meta-analyzed [35,41], because biochemical improvement, the only clinical outcome adjudicated by both studies, occurred in only one of them [35]. One study of AIC was ineligible because it was the only study examining the complex multi-agent regimen referenced above [23], and one study of ASC was ineligible because no clinical outcome was reported for both treatment groups [33].

There were notable studies of each overlap syndrome that did not meet inclusion criteria for our systematic review. The absence of $\geq 2$ distinct treatment groups $[2,3,5,18,21,32,34,36-38,40,42,76]$ or the failure to stratify clinical outcomes by treatment group $[9,19,20,46,58]$ were the most common reasons for exclusion. Three studies examining the impact of MMF as second-line therapy for AIH-PBC [16], AIH-PSC [15], AIC [15], and ASC [15,45] were also excluded. We included the larger and more recent [6] of two AIH-PBC studies by Chazouilleres et al. [4,6], given that their patient cohorts overlapped substantially. Furthermore, a retrospective cohort study of six patients with AIH-PSC was excluded for the lack of sufficient detail in its reported clinical outcomes [39]. Lastly, PBC-PSC and AIH-PBC-PSC are not represented in the systematic review because only case series $[48,50,55]$ and case reports $[47,49,51-54]$ of these syndromes were identified in our literature search.

\subsection{Characteristics of Included Studies}

The characteristics of individual studies in our systematic review are displayed in Table 1. The meta-analysis portion of our review includes 17 studies of AIH-PBC, comprising a total of 402 patients followed at medical centers in France $[6-8,10,65]$, China $[11,66,68,69,73]$, Turkey $[10,25,57,65]$, Sweden [10,22,65], South Korea [70], Japan [71,74,75], Tunisia [64,72], Italy [10,65], and the US [10,13,65, 67]. These studies ranged in sample size from five to 88 patients, and in mean or median follow-up time from 10 to 119 months. The mean or median age of patients in each study ranged from 38 to 56 years, and $82.5 \%$ of patients with AIH-PBC across all studies were female. Twelve out of the 17 studies defined AIH-PBC using the Paris criteria [4]. All 17 studies reported biochemical improvement as a clinical outcome, although in only eight studies did this encompass cholestatic (e.g., AP or GGT) as well as hepatocellular (e.g., alanine aminotransferase [ALT] or aspartate aminotransferase [AST]) markers. Furthermore, six studies provided no a priori definition of biochemical improvement as an endpoint. Four out of the 17 studies reported improvement in histologic activity $[6,57,64,75]$ but because only one of them reported it for $\geq 2$ treatment groups [6], this outcome could not be meta-analyzed. Symptomatic improvement, non-progression of liver fibrosis, and transplant-free survival were reported in three, four, and 10 of the 17 studies, respectively. 
Table 1. Studies included in systematic review.

\begin{tabular}{|c|c|c|c|c|c|c|}
\hline Study & Design & Population & $N$ & Treatments & Outcomes & $\begin{array}{c}\text { Follow-Up } \\
\text { (months) }\end{array}$ \\
\hline \multicolumn{7}{|l|}{ AIH-PBC } \\
\hline $\begin{array}{l}\text { Chazouillères } \\
2006 \text { [6] }\end{array}$ & $\begin{array}{l}\text { Retrospective } \\
\text { cohort study }\end{array}$ & $\begin{array}{l}\text { AIH-PBC (Paris criteria) [4]. } \\
\text { Median age } 41 ; 88 \% \text { female }\end{array}$ & 17 & $\begin{array}{ll}- & \text { UDCA } 13-15 \mathrm{mg} / \mathrm{kg} / \mathrm{d}(\mathrm{n}=11) \\
- & \text { UDCA } 13-15 \mathrm{mg} / \mathrm{kg} / \mathrm{d}+ \\
\text { prednisolone } 0.5 \mathrm{mg} / \mathrm{kg} / \mathrm{d} \pm \mathrm{AZA} \\
50-100 \mathrm{mg} / \mathrm{d}(\mathrm{n}=6)\end{array}$ & $\begin{array}{l}\text { Complete biochemical } \\
\text { response (ALT }<2 \times \text { ULN, } \\
\operatorname{IgG}<16 \mathrm{~g} / \mathrm{L}) ; \text { improved } \\
\text { histologic activity; fibrosis } \\
\text { non-progression; TFS }\end{array}$ & Median 90 \\
\hline Efe 2014 [65] & $\begin{array}{l}\text { Retrospective } \\
\text { cohort study }\end{array}$ & $\begin{array}{l}\text { AIH-PBC (Paris criteria) [4]. } \\
\text { Mean age 50; } 89 \% \text { female }\end{array}$ & 19 & $\begin{array}{ll}- & \text { UDCA } 12-15 \mathrm{mg} / \mathrm{kg} / \mathrm{d}(\mathrm{n}=1) \\
- & \text { UDCA } 12-15 \mathrm{mg} / \mathrm{kg} / \mathrm{d}+\text { prednisone } \\
30-60 \mathrm{mg} / \mathrm{d} \pm \text { AZA } 50-150 \mathrm{mg} / \mathrm{d} \\
(\mathrm{n}=18)\end{array}$ & $\begin{array}{l}\text { Biochemical remission } \\
\text { (normalization or }>40 \% \\
\text { reduction in AP at } 1 \text { year, } \\
\text { normalization of } \\
\text { transaminases); TFS }\end{array}$ & Mean 50 \\
\hline Fan 2018 [66] & $\begin{array}{l}\text { Prospective } \\
\text { cohort study }\end{array}$ & $\begin{array}{l}\text { AIH-PBC (Paris criteria) [4]. } \\
\text { Median age } 60 \text { (UDCA group), } \\
48 \text { (combination therapy } \\
\text { group); } 89 \% \text { female }\end{array}$ & 28 & $\begin{array}{ll}- & \text { UDCA } 13-15 \mathrm{mg} / \mathrm{kg} / \mathrm{d}(\mathrm{n}=14) \\
\text { - } & \text { UDCA } 13-15 \mathrm{mg} / \mathrm{kg} / \mathrm{d}+ \\
\text { methylprednisolone } 12-40 \mathrm{mg} / \mathrm{d} \pm \\
\text { AZA } 50-100 \mathrm{mg} / \mathrm{d} \text { or MMF (dose not } \\
\text { reported, } \mathrm{n}=14)\end{array}$ & $\begin{array}{l}\text { Symptomatic improvement; } \\
\text { biochemical remission of } \\
\text { AIH features (normalization } \\
\text { of ALT, AST, and IgG at } \\
1 \text { year); TFS }\end{array}$ & Median 18 \\
\hline Gunsar 2002 [57] & $\begin{array}{l}\text { Retrospective } \\
\text { cohort study }\end{array}$ & $\begin{array}{l}\text { AIH-PBC (histologic, serologic, } \\
\text { and biochemical features of } \\
\text { both diseases). Median age } \\
44 \text { years, } 90 \% \text { female }\end{array}$ & $16^{*}$ & $\begin{array}{ll}- & \text { UDCA } 13 \mathrm{mg} / \mathrm{kg} / \mathrm{d}(\mathrm{n}=12) \\
- & \mathrm{UDCA} 13 \mathrm{mg} / \mathrm{kg} / \mathrm{d}+\text { prednisolone } \\
& 0.5 \mathrm{mg} / \mathrm{kg} / \mathrm{d}(\mathrm{n}=4)\end{array}$ & $\begin{array}{c}\text { Biochemical improvement }^{\dagger} \\
\text { (Significant decrease in ALT, } \\
\text { AST, AP, and globulin levels); } \\
\text { improved histologic } \\
\text { activity; TFS }\end{array}$ & Median 28 \\
\hline Heurgue 2007 [8] & $\begin{array}{l}\text { Retrospective } \\
\text { cohort study }\end{array}$ & $\begin{array}{l}\text { AIH-PBC (Paris criteria) [4]. } \\
\text { Median age } 44 ; 87 \% \text { female }\end{array}$ & 15 & $\begin{array}{ll}- & \text { UDCA 11-14.7 mg/kg/d }(\mathrm{n}=6) \\
- & \text { Corticosteroids } 0.5-1 \mathrm{mg} / \mathrm{kg} / \mathrm{d} \pm \\
& \text { AZA 1.1-2.0 mg/kg/d }(\mathrm{n}=5) \\
\text { - } & \text { UDCA 11-14.7 mg/kg/d + } \\
& \text { corticosteroids } 0.5-1 \mathrm{mg} / \mathrm{kg} / \mathrm{d} \pm \text { AZA } \\
& 1.1-2.0 \mathrm{mg} / \mathrm{kg} / \mathrm{d}(\mathrm{n}=4)\end{array}$ & $\begin{array}{l}\text { Complete biochemical } \\
\text { response (ALT decreased to } \\
<2 \times \text { ULN, AP, and GGT } \\
\text { normalized); TFS }\end{array}$ & Median 60 \\
\hline
\end{tabular}


Table 1. Cont.

\begin{tabular}{|c|c|c|c|c|c|c|}
\hline Study & Design & Population & $N$ & Treatments & Outcomes & $\begin{array}{l}\text { Follow-Up } \\
\text { (months) }\end{array}$ \\
\hline \multicolumn{7}{|l|}{ АIH-PBC } \\
\hline Joshi 2002 [8] & $\begin{array}{l}\text { Retrospective } \\
\text { cohort study }\end{array}$ & $\begin{array}{l}\text { AIH-PBC (Paris criteria) [4]. } \\
\text { Median age } 46 ; 94 \% \text { female }\end{array}$ & 16 & $\begin{array}{ll}- & \text { UDCA } 13-15 \mathrm{mg} / \mathrm{kg} / \mathrm{d}(\mathrm{n}=12) \\
- & \text { Placebo }(\mathrm{n}=4)\end{array}$ & $\begin{array}{l}\text { Improved histologic activity } \\
\text { ("standardized scoring } \\
\text { system for lobular } \\
\text { inflammation", } \\
\text { not otherwise specified) }\end{array}$ & Median 84 \\
\hline Levy 2014 [67] & $\begin{array}{l}\text { Retrospective } \\
\text { cohort study }\end{array}$ & $\begin{array}{c}\text { AIH-PBC (Paris criteria) [4]. } \\
\text { Median age } 50-55 ; 92 \% \text { female }\end{array}$ & 39 & $\begin{array}{ll}- & \text { UDCA } 14-15 \mathrm{mg} / \mathrm{kg} / \mathrm{d}(\mathrm{n}=18) \\
\text { - } & \text { UDCA } 14-15 \mathrm{mg} / \mathrm{kg} / \mathrm{d}+\text { AZA or } \\
\text { MMF or prednisone (doses not } \\
\text { reported, } \mathrm{n}=21)\end{array}$ & $\begin{array}{l}\text { Complete biochemical } \\
\text { response }{ }^{+} \text {("normalization of } \\
\text { liver biochemistries", } \\
\text { not otherwise specified) }\end{array}$ & Median 38 \\
\hline $\begin{array}{c}\text { Lindgren } 2009 \\
{[22]}\end{array}$ & $\begin{array}{l}\text { Retrospective } \\
\text { cohort study }\end{array}$ & $\begin{array}{l}\text { AIH-PBC (AIH-IAIHG } \\
\quad \text { revised score [27]; } \\
\text { PBC-histology, AMA+). } \\
\text { Mean age 56; 88\% female }\end{array}$ & 25 & $\begin{array}{ll}\text { - } & \text { UDCA (dose not reported, } \mathrm{n}=18) \\
\text { - } & \text { Corticosteroids } \pm \text { aza (doses not } \\
& \text { reported, } \mathrm{n}=15)^{\ddagger}\end{array}$ & $\begin{array}{l}\text { Biochemical remission } \\
\text { (normalization } \\
\text { of transaminases) }\end{array}$ & Mean 168 \\
\hline Liu 2014 [69] & $\begin{array}{l}\text { Retrospective } \\
\text { cohort study }\end{array}$ & $\begin{array}{l}\text { AIH-PBC (AIH-IAIHG } \\
\text { simplified score [28]; } \\
\text { PBC-Paris criteria) [4]. } \\
\text { Median age } 53 ; 86 \% \text { female }\end{array}$ & $7 \S$ & $\begin{array}{l}\text { - } \quad \text { UDCA } 13-15 \mathrm{mg} / \mathrm{kg} / \mathrm{d}(\mathrm{n}=6) \\
\text { - } \quad \text { UDCA } 13-15 \mathrm{mg} / \mathrm{kg} / \mathrm{d}+\text { prednisone } \\
10-60 \mathrm{mg} / \mathrm{d} \pm \text { AZA } 50 \mathrm{mg} / \mathrm{d}(\mathrm{n}=1)\end{array}$ & $\begin{array}{c}\text { "Complete response" = } \\
\text { histologic improvement or } \\
\text { biochemical response }(\text { ALT }< \\
2 \times \mathrm{ULN}, \operatorname{IgG}<15.6 \mathrm{~g} / \mathrm{L})\end{array}$ & Range 9-48 \\
\hline Ozaslan 2010 [25] & $\begin{array}{l}\text { Retrospective } \\
\text { cohort study }\end{array}$ & $\begin{array}{l}\text { AIH-PBC (Paris criteria) [4]. } \\
\text { Median age } 44 ; 92 \% \text { female }\end{array}$ & 12 & $\begin{array}{ll}- & \text { UDCA (dose not reported, } \mathrm{n}=3) \\
- & \text { UDCA + prednisolone or AZA } \\
& (\text { doses not reported, } \mathrm{n}=9)\end{array}$ & $\begin{array}{l}\text { Symptom resolution; } \\
\text { complete biochemical } \\
\text { remission (ALT and AST < } \\
2 \times \text { ULN, Tbili, and gamma } \\
\text { globulin normalization); TFS }\end{array}$ & Median 32 \\
\hline Ozaslan 2014 [25] & $\begin{array}{l}\text { Retrospective } \\
\text { cohort study }\end{array}$ & $\begin{array}{l}\text { AIH-PBC (Paris criteria) [4]. } \\
\text { Median age } 48 ; 84 \% \text { female }\end{array}$ & 88 & $\begin{array}{l}\text { - } \quad \text { UDCA } 13-15 \mathrm{mg} / \mathrm{kg} / \mathrm{d}(\mathrm{n}=30) \\
\text { - } \quad \text { UDCA } 13-15 \mathrm{mg} / \mathrm{kg} / \mathrm{d}+\text { prednisone } \\
30-60 \mathrm{mg} / \mathrm{d} \pm \text { AZA } 50-150 \mathrm{mg} / \mathrm{d} \\
(\mathrm{n}=67){ }^{\#}\end{array}$ & $\begin{array}{l}\text { Biochemical remission } \\
\text { (normalization or }>40 \% \\
\text { reduction in AP at } 1 \text { year, } \\
\text { normalization of } \\
\text { transaminases); fibrosis } \\
\text { non-progression }\end{array}$ & Mean 66 \\
\hline
\end{tabular}


Table 1. Cont

\begin{tabular}{|c|c|c|c|c|c|c|}
\hline Study & Design & Population & $N$ & Treatments & Outcomes & $\begin{array}{l}\text { Follow-Up } \\
\text { (months) }\end{array}$ \\
\hline \multicolumn{7}{|l|}{ АIH-PBC } \\
\hline Park 2015 [10] & $\begin{array}{l}\text { Retrospective } \\
\text { cohort study }\end{array}$ & $\begin{array}{l}\text { AIH-PBC (Paris criteria) [4]. } \\
\text { Median age } 49 ; 100 \% \text { female }\end{array}$ & $7^{* *}$ & $\begin{array}{ll}\text { - } & \text { UDCA (dose not reported, } \mathrm{n}=4) \\
\text { - } & \text { UDCA + corticosteroids (doses not } \\
& \text { reported, } \mathrm{n}=3 \text { ) }\end{array}$ & $\begin{array}{l}\text { Biochemical remission (For } \\
\text { UDCA + corticosteroid } \\
\text { group: Normalization of } \\
\text { transaminases, Tbili, IgG. For } \\
\text { UDCA group: AP }<3 \times \text { ULN, } \\
\text { AST }<2 \times \text { ULN, Tbili } \leq 1 \\
\text { mg/dL within } 1 \text { year) }\end{array}$ & Median 70 \\
\hline Poupon 2006 [70] & $\begin{array}{l}\text { Retrospective } \\
\text { cohort study }\end{array}$ & $\begin{array}{l}\text { AIH-PBC (Paris criteria) [4]. } \\
\text { Mean age } 46 ; 100 \% \text { female }\end{array}$ & 12 & $\begin{array}{ll}- & \text { UDCA } 12-15 \mathrm{mg} / \mathrm{kg} / \mathrm{d}(\mathrm{n}=2) \\
\text { - } & \text { UDCA } 12-15 \mathrm{mg} / \mathrm{kg} / \mathrm{d}+\text { prednisone } \\
0.5 \mathrm{mg} / \mathrm{kg} / \mathrm{d} \pm \mathrm{AZA} 1.5 \mathrm{mg} / \mathrm{kg} / \mathrm{d} \\
(\mathrm{n}=10)\end{array}$ & $\begin{array}{l}\text { Sustained biochemical } \\
\text { remission }(\mathrm{ALT} \leq 2 \times \mathrm{ULN} \\
\text { Tbili }<20 \mathrm{~mol} / \mathrm{L}) \text {; TFS }\end{array}$ & Not reported \\
\hline Saito 2006 [71] & $\begin{array}{l}\text { Retrospective } \\
\text { cohort study }\end{array}$ & $\begin{array}{l}\text { AIH-PBC (Paris criteria) [4]. } \\
\text { Median age } 55 ; 80 \% \text { female }\end{array}$ & 10 & $\begin{array}{ll}- & \text { UDCA 300-600 mg/d }(\mathrm{n}=3) \\
- & \text { Prednisolone } 30 \mathrm{mg} / \mathrm{d}(\mathrm{n}=1) \\
- & \text { UDCA 300-600 mg/d + prednisolone } \\
& 4-30 \mathrm{mg} / \mathrm{d} \pm \text { AZA (dose not } \\
& \text { reported, } \mathrm{n}=6)\end{array}$ & $\begin{array}{c}\text { Biochemical response }^{+} \\
(\mathrm{ALT}<2 \times \mathrm{ULN}) ; \mathrm{TFS}\end{array}$ & Median 84 \\
\hline Wu 2006 [73] & $\begin{array}{l}\text { Retrospective } \\
\text { cohort study }\end{array}$ & $\begin{array}{c}\text { AIH-PBC (AIH-IAIHG } \\
\text { revised score [27]; } \\
\text { PBC_-AASLD guidelines) [77]. } \\
\text { Mean age 51; gender } \\
\text { not reported }\end{array}$ & 12 & $\begin{array}{ll}\text { - } & \text { UDCA } 13-15 \mathrm{mg} / \mathrm{kg} / \mathrm{d}(\mathrm{n}=3) \\
- & \text { Prednisone } 50 \mathrm{mg} / \mathrm{d}(\mathrm{n}=3) \\
- & \text { UDCA } 13-15 \mathrm{mg} / \mathrm{kg} / \mathrm{d}+\text { prednisone } \\
& 50 \mathrm{mg} / \mathrm{d}(\mathrm{n}=6)\end{array}$ & $\begin{array}{l}\text { Complete biochemical } \\
\text { remission }{ }^{\dagger} \text { (transaminases }< \\
2 \times \mathrm{ULN}_{\text {, significant decrease }} \\
\text { in AP and GGT) }\end{array}$ & Not reported \\
\hline Yang 2016 [73] & $\begin{array}{l}\text { Retrospective } \\
\text { cohort study }\end{array}$ & 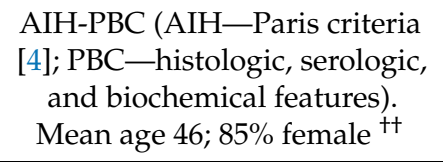 & 35 㧊 & $\begin{array}{ll}- & \text { UDCA } 13-15 \mathrm{mg} / \mathrm{kg} / \mathrm{d}(\mathrm{n}=8) \\
- & \text { UDCA 13-15 mg/kg/d + } \\
& \text { prednisolone } 15-50 \mathrm{mg} / \mathrm{d}(\mathrm{n}=27)\end{array}$ & $\begin{array}{c}\text { Biochemical remission } \\
\text { (Paris-I criteria: } \mathrm{AP}<3 \times \\
\text { ULN, AST }<2 \times \\
\text { ULN, Tbili normalization) }\end{array}$ & Median 38 \\
\hline $\begin{array}{c}\text { Yokokawa } 2010 \\
{[74]}\end{array}$ & $\begin{array}{l}\text { Retrospective } \\
\text { cohort study }\end{array}$ & $\begin{array}{l}\text { AIH-PBC (Paris criteria) [4]. } \\
\text { Mean age 56; 88\% female }\end{array}$ & 16 & $\begin{array}{ll}- & \text { UDCA } 300-600 \mathrm{mg} / \mathrm{d}(\mathrm{n}=2) \\
- & \text { Prednisolone } 30 \mathrm{mg} / \mathrm{d}(\mathrm{n}=1) \\
- & \text { UDCA 300-600 mg/d + prednisolone } \\
& 10-40 \mathrm{mg} / \mathrm{d} \pm \mathrm{AZA}(\text { dose not } \\
& \text { reported, } \mathrm{n}=13)\end{array}$ & $\begin{array}{c}\text { Biochemical remission }^{\dagger} \\
\text { (normalization of ALT, AP); } \\
\text { TFS }\end{array}$ & Median 119 \\
\hline
\end{tabular}


Table 1. Cont

\begin{tabular}{|c|c|c|c|c|c|c|}
\hline Study & Design & Population & $N$ & Treatments & Outcomes & $\begin{array}{c}\text { Follow-Up } \\
\text { (months) }\end{array}$ \\
\hline \multicolumn{7}{|l|}{ АIH-PBC } \\
\hline Yoshioka 2014 [75] & $\begin{array}{l}\text { Retrospective } \\
\text { cohort study }\end{array}$ & $\begin{array}{l}\text { AIH-PBC (biochemical, } \\
\text { serologic, and histologic } \\
\text { features of both diseases) [75]. } \\
\text { Median age } 55 ; 93 \% \text { female }\end{array}$ & 28 & $\begin{array}{l}\text { UDCA (dose not reported, } \mathrm{n}=8 \text { ) } \\
\text { UDCA (dose not reported) }+ \\
\text { corticosteroids 30mg/d }(\mathrm{n}=20 \text { ) }\end{array}$ & $\begin{array}{l}\text { Biochemical remission } \\
\text { (normalization of } \\
\text { transaminases); improved } \\
\text { histologic activity (Ludwig) } \\
\text { [78]; improved piecemeal } \\
\text { necrosis (undefined); fibrosis } \\
\text { non-progression; TFS }\end{array}$ & Median 94 \\
\hline Liu 2014 [68] & $\begin{array}{l}\text { Prospective } \\
\text { cohort study }\end{array}$ & $\begin{array}{l}\text { AIH-PBC (biochemical \& } \\
\text { histologic features of both } \\
\text { diseases). Mean age 56; } \\
33 \% \text { female }\end{array}$ & 43 & $\begin{array}{ll}- & \text { UDCA } 13-15 \mathrm{mg} / \mathrm{kg} / \mathrm{d}(\mathrm{n}=16) \\
- & \text { Prednisone } 50 \mathrm{mg} / \mathrm{d}(\mathrm{n}=13) \\
- & \text { UDCA } 13-15 \mathrm{mg} / \mathrm{kg} / \mathrm{d}+\text { prednisone } \\
& 50 \mathrm{mg} / \mathrm{d}(\mathrm{n}=14)\end{array}$ & $\begin{array}{l}\text { Complete biochemical } \\
\text { remission }{ }^{\dagger} \text { (undefined) }\end{array}$ & Mean 10 \\
\hline Cheikh 2003 [64] & $\begin{array}{l}\text { Retrospective } \\
\text { cohort study }\end{array}$ & $\begin{array}{l}\text { AIH-PBC (Paris criteria) [4]. } \\
\text { Median age } 38 ; 100 \% \text { female }\end{array}$ & 5 & $\begin{array}{l}\text { UDCA } 13-15 \mathrm{mg} / \mathrm{kg} / \mathrm{d}(\mathrm{n}=3) \\
\text { Prednisone } 30 \mathrm{mg} / \mathrm{d}+\text { AZA } 1-2 \\
\mathrm{mg} / \mathrm{kg} / \mathrm{d}(\mathrm{n}=2)\end{array}$ & $\begin{array}{l}\text { Symptomatic improvement; } \\
\text { complete biochemical } \\
\text { response (normalization of } \\
\text { ALT, AP, GGT, and Tbili); } \\
\text { improved histologic activity; } \\
\text { fibrosis non-progression }\end{array}$ & Mean 17 \\
\hline Serghini 2012 [72] & $\begin{array}{l}\text { Retrospective } \\
\text { cohort study }\end{array}$ & $\begin{array}{l}\text { AIH-PBC (Paris criteria) [4]. } \\
\text { Mean age } 53 ; 100 \% \text { female }\end{array}$ & 5 & $\begin{array}{ll} & \text { UDCA } 15 \mathrm{mg} / \mathrm{kg} / \mathrm{d}+\text { corticosteroids } \\
30 \mathrm{mg} / \mathrm{d}+\text { AZA } 50 \mathrm{mg} / \mathrm{d}(\mathrm{n}=4) \\
\text { - } \quad \text { Corticosteroids } 30 \mathrm{mg} / \mathrm{d}+\text { AZA } 50 \\
\mathrm{mg} / \mathrm{d}(\mathrm{n}=1)\end{array}$ & $\begin{array}{l}\text { Complete biochemical } \\
\text { response }(\text { ALT }<2 \times \text { ULN, } \\
\text { normalization of AP } \\
\text { and GGT) }\end{array}$ & Median 11 \\
\hline Luth 2009 [35] & $\begin{array}{l}\text { Retrospective } \\
\text { cohort study }\end{array}$ & $\begin{array}{l}\text { AIH-PSC (AIH_-IAHG revised } \\
\text { score [27]; PSC_-histologic or } \\
\text { cholangiographic features). } \\
\text { Mean age } 34 ; 19 \% \text { female }\end{array}$ & 16 & $\begin{array}{ll}\text { - } & \text { Corticosteroids } \pm \text { aza (doses not } \\
& \text { reported, } \mathrm{n}=10) \\
\text { - } & \text { UDCA }+ \text { corticosteroids } \pm \text { AZA } \\
& (\text { doses not reported }, \mathrm{n}=6)\end{array}$ & $\begin{array}{l}\text { Biochemical response }^{+} \\
\text {(Improvement in ALT at } \\
6 \text { months) }\end{array}$ & Median 144 \\
\hline McNair 1998 [41] & $\begin{array}{l}\text { Prospective } \\
\text { cohort study }\end{array}$ & $\begin{array}{l}\text { AIH-PSC (AIH_definite by } \\
\text { original IAIHG score [26]; } \\
\text { PSC-positive cholangiogram). } \\
\text { Median age } 20 ; 20 \% \text { female }\end{array}$ & 5 & $\begin{array}{ll}\text { - } & \text { Prednisolone } 15-80 \mathrm{mg} / \mathrm{d}+\mathrm{AZA} \\
50-100 \mathrm{mg} / \mathrm{d}(\mathrm{n}=3) \\
\text { - } \quad \text { UDCA } 300 \mathrm{mg} 1-2 \mathrm{x} / \mathrm{d}+ \\
\text { prednisolone 20-30 mg/d + AZA } \\
75-150 \mathrm{mg} / \mathrm{d}(\mathrm{n}=2)\end{array}$ & $\begin{array}{l}\text { Symptomatic improvement; } \\
\text { improved histologic activity; } \\
\text { fibrosis non-progression } \\
\text { (Batts \& Ludwig) [79] }\end{array}$ & Median 84 \\
\hline
\end{tabular}


Table 1. Cont

\begin{tabular}{|c|c|c|c|c|c|c|}
\hline Study & Design & Population & $N$ & Treatments & Outcomes & $\begin{array}{l}\text { Follow-Up } \\
\text { (months) }\end{array}$ \\
\hline \multicolumn{7}{|l|}{$A I C$} \\
\hline Czaja 2000 [24] & $\begin{array}{l}\text { Prospective } \\
\text { cohort study }\end{array}$ & $\begin{array}{l}\text { AIC (serologic and biochemical } \\
\text { features of AIH; biochemical or } \\
\text { histologic features of PBC but } \\
\text { AMA). Mean age } 46 ; \\
\text { 85\% female }\end{array}$ & 20 & $\begin{array}{ll}- & \text { UDCA } 13-15 \mathrm{mg} / \mathrm{kg} / \mathrm{d}(\mathrm{n}=8) \\
- & \text { Prednisone } \pm \text { AZA (doses not } \\
& \text { reported, } \mathrm{n}=8)\end{array}$ & $\begin{array}{l}\text { Biochemical remission (per } \\
\text { Czaja 1991) [80]; improved } \\
\text { histologic activity (criteria by } \\
\text { Ishak et al.) [81] }\end{array}$ & Not reported \\
\hline Campos 2017 [23] & $\begin{array}{l}\text { Prospective } \\
\text { cohort study }\end{array}$ & $\begin{array}{l}\text { AIC (biochemical features of } \\
\text { AIH and PBC, histologic } \\
\text { features of PBC, AMA-). Mean } \\
\text { age 28.5; } 100 \% \text { female }\end{array}$ & 2 & 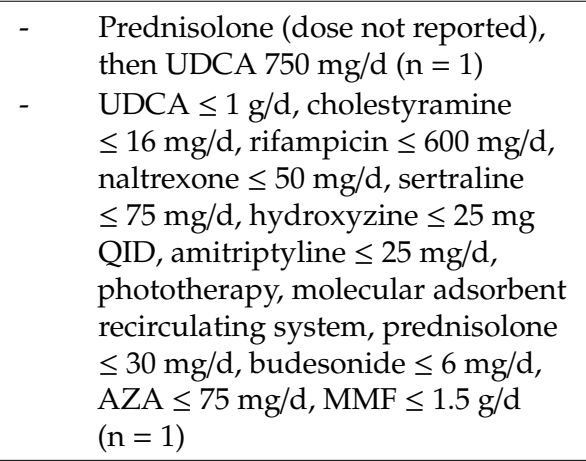 & $\begin{array}{l}\text { Symptomatic improvement; } \\
\text { biochemical remission }{ }^{+} \\
\text {(normalization of ALT, AST, } \\
\text { AP, and GGT); TFS }\end{array}$ & Not reported \\
\hline $\begin{array}{c}\text { Lindgren } 2009 \\
{[22]}\end{array}$ & $\begin{array}{l}\text { Retrospective } \\
\text { cohort study }\end{array}$ & $\begin{array}{l}\text { AIC (biochemical \& histologic } \\
\text { features of PBC, ANA, or } \\
\text { ASMA+, AMA-). Mean age } 51 ; \\
88 \% \text { female }\end{array}$ & $4 \S \S$ & $\begin{array}{ll}- & \text { UDCA (dose not reported, } \mathrm{n}=1) \\
\text { - } & \text { Corticosteroids } \pm \text { aza (doses not } \\
& \text { reported, } \mathrm{n}=3)\end{array}$ & $\begin{array}{l}\text { Biochemical remission } \\
\text { (normalization of } \\
\text { transaminases) }\end{array}$ & Mean 127 \\
\hline Smolka 2016 [44] & $\begin{array}{l}\text { Retrospective } \\
\text { cohort study }\end{array}$ & $\begin{array}{l}\text { ASC (probable or definite AIH } \\
\text { by simplified IAIHG score } \\
\text { modified for children [82]; } \\
\text { positive cholangiogram). } \\
\text { Median age } 14 ; 55 \% \text { female }\end{array}$ & 11 & $\begin{array}{ll}- & \text { UDCA } 15-20 \mathrm{mg} / \mathrm{kg} / \mathrm{d}(\mathrm{n}=2) \\
- & \text { UDCA } 15-20 \mathrm{mg} / \mathrm{kg} / \mathrm{d}+\text { prednisone } \\
& 1-2 \mathrm{mg} / \mathrm{kg} / \mathrm{d}+\mathrm{AZA} 1-2 \mathrm{mg} / \mathrm{kg} / \mathrm{d}(\mathrm{n} \\
& =9)\end{array}$ & $\begin{array}{l}\text { Biochemical remission } \\
\quad \text { (undefined); TFS }\end{array}$ & Median 144 \\
\hline
\end{tabular}


Table 1. Cont.

\begin{tabular}{|c|c|c|c|c|c|c|}
\hline Study & Design & Population & $N$ & Treatments & Outcomes & $\begin{array}{l}\text { Follow-Up } \\
\text { (months) }\end{array}$ \\
\hline \multicolumn{7}{|l|}{$A I C$} \\
\hline Ferrari 2018 [43] & $\begin{array}{l}\text { Retrospective } \\
\text { cohort study }\end{array}$ & $\begin{array}{l}\text { ASC (biochemical and } \\
\quad \text { histologic and/or } \\
\text { cholangiographic features of } \\
\text { PSC; AIH features on IAIHG } \\
\text { revised score) [27]. Mean age } \\
\text { 9.9; gender not reported }\end{array}$ & $14^{\mathrm{III}}$ & $\begin{array}{ll}- & \text { UDCA } 15-20 \mathrm{mg} / \mathrm{kg} / \mathrm{d}(\mathrm{n}=2) \\
\text { - } & \text { UDCA } 15-20 \mathrm{mg} / \mathrm{kg} / \mathrm{d}+\text { prednisone } \\
1 \mathrm{mg} / \mathrm{kg} / \mathrm{d}+\mathrm{AZA} 1.5-2 \mathrm{mg} / \mathrm{kg} / \mathrm{d} \\
(\mathrm{n}=12)\end{array}$ & $\begin{array}{l}\text { Biochemical remission }{ }^{\dagger} \\
\quad \text { (undefined) }\end{array}$ & Median 79 \\
\hline Gregorio 2001 [33] & $\begin{array}{l}\text { Prospective } \\
\text { cohort study }\end{array}$ & $\begin{array}{l}\text { ASC (probable or definite AIH } \\
\text { by IAIHG revised score [27]; } \\
\text { positive cholangiogram. } \\
\text { Median age } 11.8 ; 56 \% \text { female }\end{array}$ & $26^{\# \#}$ & $\begin{array}{ll}- & \text { UDCA (dose not reported, } \mathrm{n}=3 \text { ) } \\
\text { - } & \text { Prednisolone } 2 \mathrm{mg} / \mathrm{kg} / \mathrm{d} \pm \text { UDCA } \\
\text { (dose not reported) } \pm \text { AZA } 1-2 \\
\mathrm{mg} / \mathrm{kg} / \mathrm{d}(\mathrm{n}=23)\end{array}$ & $\begin{array}{c}\text { Biochemical remission } \\
\text { (normalization of liver } \\
\text { function tests); improved } \\
\text { histologic activity } \\
\text { (inflammatory activity index } \\
\text { scored 0-12, } \\
\text { Incorporating portal tract } \\
\text { inflammation, lobular } \\
\text { activity, and piecemeal } \\
\text { necrosis) [33] }\end{array}$ & Median 72 \\
\hline
\end{tabular}

Transplant-free survival is expressed as the raw fraction of study participants who did not experience death or liver transplant by the end of follow-up. Fibrosis and histologic activity were assessed using the METAVIR scoring system $(\mathrm{A} 0=$ absent, $\mathrm{A} 1=$ mild, $\mathrm{A} 2=$ moderate, and $\mathrm{A} 3=$ severe histologic activity; F0 = no fibrosis, $\mathrm{F} 1=$ portal fibrosis without septa, $\mathrm{F} 2$ = few septa, F3 = numerous septa without cirrhosis, and F4 = cirrhosis) [83] except where otherwise noted. In treatment regimens comprising "corticosteroids", the authors did not specify which corticosteroid(s) were used. * Excluded 1 patient lost to follow-up and 3 patients who crossed between treatment groups. ${ }^{\dagger}$ Biochemical endpoint not defined a priori in Methods,

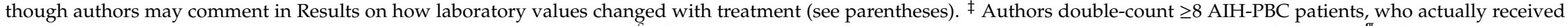
both UDCA and corticosteroids, in the UDCA and corticosteroid groups. \& Of the 10 AIH-PBC patients studied, treatment outcomes were reported for only 7 . ${ }^{\text {II }}$ Of the 5 patients who eventually received combination therapy, the 4 who received UDCA monotherapy beforehand were censored. \# Unable to exclude 9 patients from combination therapy group who crossed over from UDCA group, because authors do not distinguish their treatment outcomes from those of non-crossover patients. ${ }^{* *}$ Excluded 2 patients who crossed between treatment groups. ${ }^{++}$This percentage pertains to the total of 46 AIH-PBC patients (see below). $¥$ Of Of the 46 AIH-PBC patients studied, treatment outcomes were reported for only 35 . $\$ \$$ Of the 8 AIC groups. This percentage pertains to the total of 46 AIH-PBC patients (see below). ${ }^{+1}$ Of the 46 AIH-PBC patients studied, treatment outcomes were reported for only 35 . 88 Of the 8 AIC
patients studied, treatment outcomes were reported for only 4 . Note that $7(88 \%)$ of the total 8 patients were female. patients studied, treatment outcomes were reported for only 4 . Note that $7(88 \%)$ of the total 8 patients were female. 111 Excluded 5 patients who crossed between treatment groups. cholangitis, PSC: Primary sclerosing cholangitis, AIC: Autoimmune cholangitis, ASC: Autoimmune sclerosing cholangitis, AMA: antimitochondrial antibody, ANA: Antinuclear antibody, ASMA: Anti-smooth muscle antibody, IAIHG: International Autoimmune Hepatitis Group, AASLD: American Association for the Study of Liver Diseases, UDCA: Ursodeoxycholic acid, MMF: Mycophenolate mofetil, ALT: Alanine aminotransferase, IgG: Immunoglobulin G, Tbili: Total bilirubin, ULN: Upper limit of normal, TFS: Transplant-free survival, AST: Aspartate aminotransferase, AP: Alkaline phosphatase, GGT: Gamma-glutamyl transferase. 
Twenty-four patients with AIC were included in the meta-analysis of which four are from a study in Sweden [22] and 20 from a study in the US [24]. The former had a mean follow-up period of 127 months, while the latter did not report duration of follow-up. These patients were a mean of 47.4 years old, and $86 \%$ were female. Both studies defined AIC as biochemical and/or serologic evidence of AIH with biochemical and/or histologic evidence of PBC in the absence of anti-mitochondrial antibodies. Biochemical improvement was reported in both studies (though without an a priori definition) and encompassed only hepatocellular, not cholestatic, biomarkers. One of the two studies reported improvement in histologic activity [24], and neither study reported symptomatic improvement, fibrosis non-progression, or transplant-free survival.

Lastly, the meta-analysis includes two studies comprising 25 patients with ASC. One of these studies was conducted in the Czech Republic and followed 11 patients (six female, five male) for a median of 144 months [44]. The other, conducted in Italy, followed 14 patients for a median of 79 months and did not report their gender distribution [43]. The median ages of patients in the former and latter studies were 14 and 9.9 years, respectively. Both studies used a form of the IAIHG criteria to define the AIH component of ASC and for the PSC component, one study required biochemical cholestasis with characteristic findings on either histology or cholangiography [43], while the other study required just cholangiographic findings [44]. Both studies reported biochemical improvement but failed to define it in any detail. Neither study reported symptomatic improvement, improvement in histologic activity, fibrosis non-progression, or transplant-free survival.

\subsection{Quality of Included Studies}

The quality of individual cohort studies in our systematic review was quantified using the Newcastle-Ottawa Scale [62] as shown in Table 2. Lindgren et al. was scored separately for AIH-PBC and AIC, therefore, the effective total number of studies referred to in this paragraph is 29 rather than 28 [22]. Twenty-six out of these 29 studies-20/21 AIH-PBC studies, 2/2 AIH-PSC studies, 1/3 AIC studies, and 3/3 ASC studies-scored 7 out of 9 possible points. None of these 26 studies received the 1-2 points corresponding to comparability of patient cohorts because they did not control for possible confounders in their design or analysis. One cohort study of AIH-PBC nested within a randomized controlled trial did receive an additional point for patient cohort comparability, thereby scoring 8 points [13]. Two studies of AIC scored 6/9 points and in addition to losing 2 points for comparability of patient cohorts, one study lost a point for failing to report the duration of follow-up [23], while the other lost a point for having $>10 \%$ patients lost to follow-up (treatment outcomes were reported for only four out of eight patients, for unclear reasons) [22]. 
Table 2. Quality of studies included in systematic review as quantified by Newcastle-Ottawa Scale.

\begin{tabular}{|c|c|c|c|c|c|c|c|c|c|}
\hline Study & Selection & & & & Comparability & Exposure & & & \\
\hline & $\begin{array}{c}\text { Represent- } \\
\text { Ativeness of } \\
\text { Exposed Cohort }\end{array}$ & $\begin{array}{c}\text { Selection of } \\
\text { Non-Exposed } \\
\text { Cohort }\end{array}$ & $\begin{array}{l}\text { Ascertainment } \\
\text { of Exposure }\end{array}$ & $\begin{array}{l}\text { Demonstration that } \\
\text { Outcomes of Interest } \\
\text { Were not Present at } \\
\text { Start of Study }\end{array}$ & $\begin{array}{l}\text { Comparability of } \\
\text { Cohorts on Basis } \\
\text { of Design or } \\
\text { Analysis * }\end{array}$ & $\begin{array}{l}\text { Assessment } \\
\text { of Outcomes }\end{array}$ & $\begin{array}{l}\text { Length of } \\
\text { Follow-Up }\end{array}$ & $\begin{array}{l}\text { Adequacy of } \\
\text { Follow-Up } \ddagger\end{array}$ & Total \\
\hline Campos 2017 [23] & * & $*$ & $*$ & $*$ & & $*$ & & * & 6 \\
\hline Chazouillères 2006 [6] & * & * & * & * & & * & * & * & 7 \\
\hline Cheikh 2003 [64] & $*$ & $*$ & $*$ & $*$ & & $*$ & $*$ & $*$ & 7 \\
\hline Czaja 2000 [24] & $*$ & $*$ & $*$ & $*$ & & $*$ & $*$ & $*$ & 7 \\
\hline Efe 2014 [65] & $*$ & $*$ & $*$ & $*$ & & $*$ & $*$ & $*$ & 7 \\
\hline Fan 2018 [66] & $*$ & $*$ & $*$ & $*$ & & $*$ & $*$ & $*$ & 7 \\
\hline Ferrari 2018 [43] & $*$ & $*$ & $*$ & $*$ & & $*$ & $*$ & $*$ & 7 \\
\hline Gregorio 2001 [33] & $*$ & $*$ & $*$ & $*$ & & $*$ & $*$ & $*$ & 7 \\
\hline Gunsar 2002 [57] & $*$ & $*$ & $*$ & $*$ & & $*$ & ${ }^{*}$ & $*$ & 7 \\
\hline Heurgue 2007 [8] & $*$ & $*$ & $*$ & $*$ & & * & * & $*$ & 7 \\
\hline Joshi 2002 [8] & $*$ & $*$ & $*$ & $*$ & $*$ & $*$ & $*$ & * & 8 \\
\hline Levy 2014 [67] & $*$ & $*$ & $*$ & $*$ & & * & * & * & 7 \\
\hline \multicolumn{10}{|l|}{ Lindgren 2009 [22] } \\
\hline AIH-PBC & $*$ & $*$ & $*$ & $*$ & & $*$ & $*$ & $*$ & 7 \\
\hline AIC & $*$ & $*$ & $*$ & $*$ & & $*$ & $*$ & & 6 \\
\hline Liu 2014 [69] & $*$ & $*$ & $*$ & $*$ & & $*$ & $*$ & $*$ & 7 \\
\hline Liu 2014 [68] & $*$ & $*$ & $*$ & $*$ & & $*$ & $*$ & $*$ & 7 \\
\hline Luth 2009 [35] & * & $*$ & $*$ & $*$ & & * & $*$ & * & 7 \\
\hline McNair 1998 [41] & $*$ & $*$ & $*$ & $*$ & & $*$ & $*$ & $*$ & 7 \\
\hline Ozaslan 2010 [25] & $*$ & $*$ & $*$ & $*$ & & $*$ & $*$ & * & 7 \\
\hline Ozaslan 2014 [25] & $*$ & $*$ & $*$ & * & & * & * & $*$ & 7 \\
\hline Park 2015 [10] & $*$ & $*$ & $*$ & $*$ & & * & $*$ & $*$ & 7 \\
\hline Poupon 2006 [70] & * & * & * & * & & * & $* \S$ & * & 7 \\
\hline
\end{tabular}


Table 2. Cont.

\begin{tabular}{|c|c|c|c|c|c|c|c|c|c|}
\hline Study & Selection & & & & Comparability & Exposure & & & \\
\hline & $\begin{array}{c}\text { Represent- } \\
\text { Ativeness of } \\
\text { Exposed Cohort }\end{array}$ & $\begin{array}{c}\text { Selection of } \\
\text { Non-Exposed } \\
\text { Cohort }\end{array}$ & $\begin{array}{l}\text { Ascertainment } \\
\text { of Exposure }\end{array}$ & $\begin{array}{l}\text { Demonstration that } \\
\text { Outcomes of Interest } \\
\text { Were not Present at } \\
\text { Start of Study }\end{array}$ & $\begin{array}{c}\text { Comparability of } \\
\text { Cohorts on Basis } \\
\text { of Design or } \\
\text { Analysis * }\end{array}$ & $\begin{array}{l}\text { Assessment } \\
\text { of Outcomes }\end{array}$ & $\begin{array}{l}\text { Length of } \\
\text { Follow-Up }\end{array}$ & $\begin{array}{l}\text { Adequacy of } \\
\text { Follow-Up } \ddagger\end{array}$ & Total \\
\hline Saito 2006 [71] & $*$ & $*$ & $*$ & $*$ & & $*$ & * & * & 7 \\
\hline Serghini 2012 [72] & * & * & $*$ & * & & * & * & * & 7 \\
\hline Smolka 2016 [44] & $*$ & $*$ & $*$ & $*$ & & * & $*$ & * & 7 \\
\hline Wu 2006 [73] & $*$ & $*$ & $*$ & $*$ & & $*$ & $* \S$ & * & 7 \\
\hline Yang 2016 [73] & $*$ & $*$ & $*$ & $*$ & & $*$ & $*$ & $*$ & 7 \\
\hline Yokokawa 2010 [74] & $*$ & $*$ & $*$ & $*$ & & $*$ & $*$ & $*$ & 7 \\
\hline Yoshioka 2014 [75] & * & * & * & * & & * & * & * & 7 \\
\hline
\end{tabular}

* One point awarded for each variable that was statistically controlled for, up to a maximum of 2 points. The only point awarded was to Joshi et al., given the latter's study design (retrospective cohort study nested within a randomized controlled trial). ${ }^{\dagger}$ Criterion to gauge if follow-up time was sufficient for outcomes of interest to occur. For this analysis, 2 months, 6 months, and 5 years were considered sufficient for biochemical, histologic, or transplant-free survival outcomes, respectively. One point was awarded if the study's follow-up period was adequate for $\geq 1$ of these outcomes and if that outcome was reported. $f$ One point awarded if number of subjects lost to follow-up was small enough ( $\leq 10 \%$ in this analysis) to make attrition bias unlikely. ${ }^{\S}$ Median follow-up not specified, but several patients were followed for $\geq 2$ months and biochemical remission did occur 


\subsection{Clinical Outcomes}

\subsubsection{AIH-PBC}

When comparing combination therapy to UDCA in patients with AIH-PBC, no differences were seen in the rates of symptomatic improvement ( $\mathrm{RR}=0.75,95 \% \mathrm{CI} 0.25-2.22, p=0.60, \mathrm{I}^{2}=79 \%$ ), biochemical improvement ( $\left.\mathrm{RR}=1.30,95 \% \mathrm{CI} 0.90-1.87, p=0.16, \mathrm{I}^{2}=57 \%\right)$, non-progression of liver fibrosis $\left(\mathrm{RR}=1.40,95 \% \mathrm{CI} 0.61-3.21, p=0.42, \mathrm{I}^{2}=77 \%\right)$, or transplant-free survival $(\mathrm{RR}=1.06$, $95 \%$ CI $0.82-1.37, p=0.65, \mathrm{I}^{2}=53 \%$ ) (Figures $1-4$ ). When Fan and Levy et al. were excluded as a sensitivity analysis to simplify the combination therapy arm to UDCA + corticosteroids \pm AZA, there remained no difference in biochemical improvement between treatment groups (Figure A2). The same was observed when only studies using the Paris criteria to define AIH-PBC were included in the meta-analysis (Figure A3). When the meta-analysis was restricted to studies where biochemical improvement was defined by both hepatocellular and cholestatic biomarkers, there was a trend toward more biochemical improvement with combination therapy than with UDCA alone (RR $=1.34,95 \% \mathrm{CI}$ $0.93-1.93, p=0.12, \mathrm{I}^{2}=27 \%$ ) (Figure A4). In a sensitivity analysis including only studies with a mean or median follow-up period of $>90$ months, transplant-free survival was greater in patients receiving combination therapy than in those receiving UDCA alone (RR $=6.50,95 \%$ CI 1.47-28.83, $p=0.01$, $\mathrm{I}^{2}=0 \%$ ) (Figure A5). When comparing corticosteroids \pm AZA to UDCA for the treatment of AIH-PBC, there was no difference in the rate of biochemical improvement $(\mathrm{RR}=1.14,95 \% \mathrm{CI} 0.48-2.72, p=0.76$, $\mathrm{I}^{2}=0 \%$ ) or transplant-free survival ( $\mathrm{RR}=0.96,95 \%$ CI 0.57-1.63, $p=0.88, \mathrm{I}^{2}=5 \%$ ) (Figures 5 and 6 ). There was a non-significant trend toward superiority of combination therapy over corticosteroids \pm AZA with respect to biochemical improvement ( $\mathrm{RR}=4.00,95 \% \mathrm{CI} 0.93-17.18, p=0.06, \mathrm{I}^{2}=60 \%$ ) but not transplant-free survival ( $\mathrm{RR}=2.03,95 \%$ CI 0.28-14.91, $p=0.49, \mathrm{I}^{2}=74 \%$ ) (Figures 7 and 8 ).

Of the four studies of AIH-PBC in our systematic review that were not meta-analyzed, three are of limited analytical utility: Lindgren et al. because of overlapping treatment groups [22], and Liu et al. and Serghini et al. because neither study saw biochemical improvement in any of its participants [69,72]. The fourth study noted improved histologic activity in three of nine patients treated with UDCA $(13-15 \mathrm{mg} / \mathrm{kg} / \mathrm{d})$ versus zero of two patients treated with placebo over a median follow-up of 84 months, but this difference was not statistically significant (Table 3) [13]. 


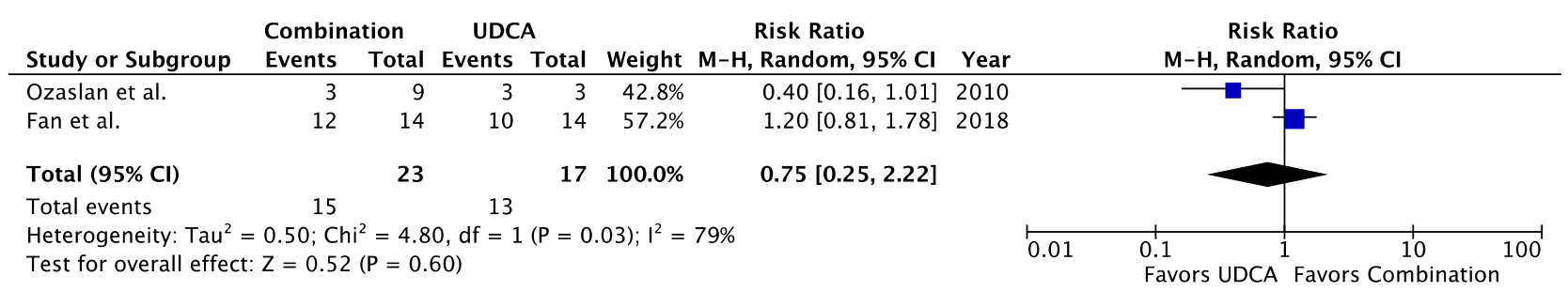

Figure 1. Symptomatic improvement in AIH-PBC patients treated with combination therapy vs. UDCA. UDCA = ursodeoxycholic acid, Combination $=$ UDCA + [corticosteroids and/or antimetabolites].

\begin{tabular}{|c|c|c|c|c|c|c|c|c|c|c|}
\hline \multirow{2}{*}{ Study or Subgroup } & \multicolumn{2}{|c|}{ Combination } & \multicolumn{2}{|c|}{ UDCA } & \multicolumn{3}{|c|}{ Risk Ratio } & & \multirow{2}{*}{\multicolumn{2}{|c|}{$\begin{array}{c}\text { Risk Ratio } \\
\text { M-H, Random, } 95 \% \mathrm{CI}\end{array}$}} \\
\hline & Events & Total & Events & Total & Weight & M-H, Random, 95\% Cl & Year & & & \\
\hline Gunsar et al. & 4 & 4 & 8 & 12 & $12.2 \%$ & $1.38[0.84,2.25]$ & 2002 & & & $F$ \\
\hline Chazouilleres et al. & 4 & 6 & 3 & 11 & $6.4 \%$ & $2.44[0.80,7.48]$ & 2006 & & & \\
\hline Saito et al. & 6 & 6 & 2 & 3 & $9.1 \%$ & $1.49[0.68,3.26]$ & 2006 & & & $=$ \\
\hline Wu et al. & 6 & 6 & 0 & 3 & $1.8 \%$ & $7.43[0.55,100.11]$ & 2006 & & & \\
\hline Poupon et al. & 8 & 10 & 2 & 2 & $11.0 \%$ & $0.93[0.51,1.69]$ & 2006 & & & - \\
\hline Heurgue et al. & 3 & 5 & 3 & 6 & $6.7 \%$ & $1.20[0.41,3.51]$ & 2007 & & & \\
\hline Ozaslan et al. & 3 & 9 & 3 & 3 & $7.8 \%$ & $0.40[0.16,1.01]$ & 2010 & & & \\
\hline Yokokawa et al. & 13 & 13 & 0 & 2 & $1.8 \%$ & $5.79[0.46,72.80]$ & 2010 & & & \\
\hline Ozaslan et al. & 49 & 67 & 19 & 30 & $14.1 \%$ & $1.15[0.85,1.57]$ & 2014 & & & $=$ \\
\hline Liu C et al. & 12 & 14 & 0 & 16 & $1.6 \%$ & $28.33[1.83,438.84]$ & 2014 & & & \\
\hline Efe et al. & 15 & 18 & 1 & 1 & $8.7 \%$ & $1.09[0.48,2.49]$ & 2014 & & & \\
\hline Levy et al. & 10 & 21 & 11 & 18 & $11.2 \%$ & $0.78[0.44,1.39]$ & 2014 & & & - \\
\hline Park et al,.. & 1 & 3 & 2 & 4 & $3.1 \%$ & $0.67[0.10,4.35]$ & 2015 & & & \\
\hline Yang et al. & 13 & 27 & 0 & 8 & $1.6 \%$ & $8.68[0.57,131.87]$ & 2016 & & & \\
\hline Fan et al. & 6 & 10 & 1 & 11 & $2.9 \%$ & $6.60[0.95,45.75]$ & 2018 & & & \\
\hline Total $(95 \% \mathrm{Cl})$ & & 219 & & 130 & $100.0 \%$ & $1.30[0.90,1.87]$ & & & & 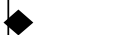 \\
\hline Total events & 153 & & 55 & & & & & & & \\
\hline $\begin{array}{l}\text { Heterogeneity: } \mathrm{Tau}^{2} \\
\text { Test for overall effec }\end{array}$ & $\begin{array}{l}0.22 ; C h \\
Z=1.40\end{array}$ & $\begin{array}{l}=32 . \\
(P=0.1\end{array}$ & $\begin{array}{l}\text { 69, } d f= \\
16)\end{array}$ & $14(P=$ & $=0.003) ;$ & & & 002 & $\begin{array}{cc}0.1 & 1 \\
\text { Favors UDCA }\end{array}$ & 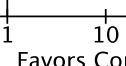 \\
\hline
\end{tabular}

Figure 2. Biochemical improvement in AIH-PBC patients treated with combination therapy vs. UDCA alone. UDCA $=$ ursodeoxycholic acid, Combination $=$ UDCA + [corticosteroids and/or antimetabolites]. 


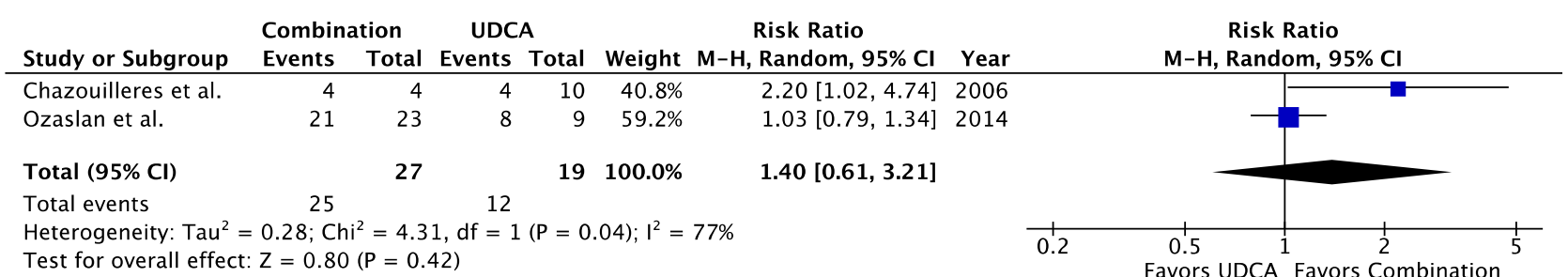

Figure 3. Non-progression of liver fibrosis in AIH-PBC patients treated with combination therapy vs. UDCA alone. UDCA = ursodeoxycholic acid, Combination = UDCA + corticosteroids \pm AZA

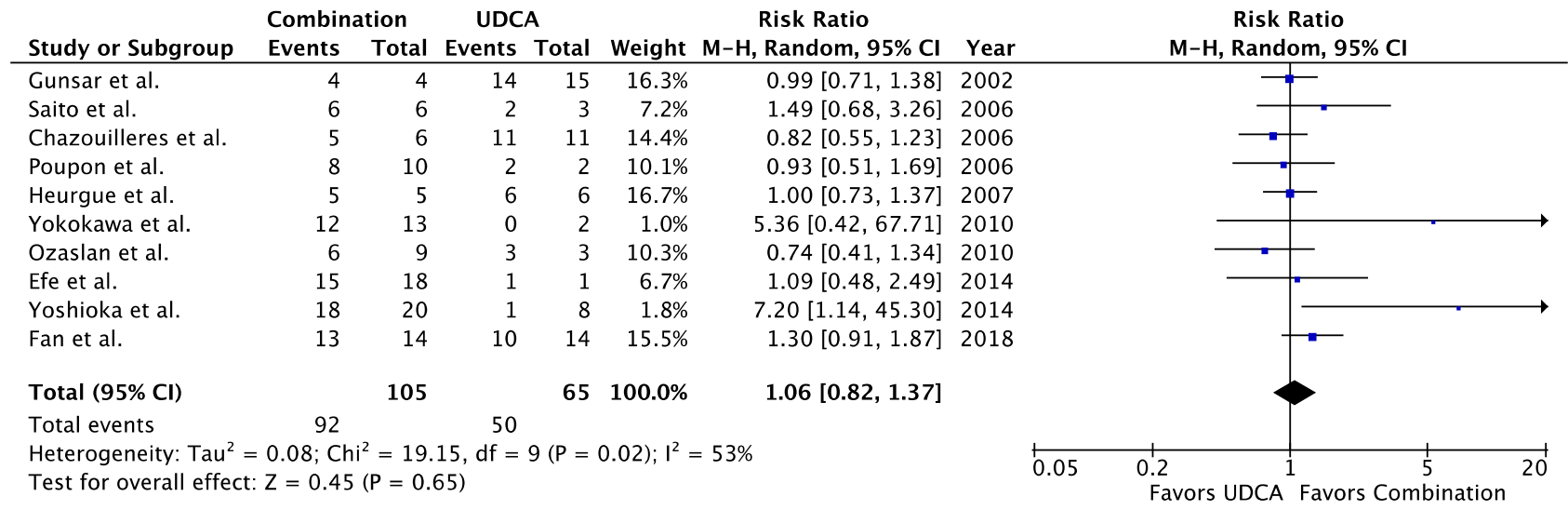

Figure 4. Transplant-free survival in AIH-PBC patients treated with combination therapy vs. UDCA alone. UDCA = ursodeoxycholic acid, Combination $=$ UDCA + [corticosteroids and/or antimetabolites]. 


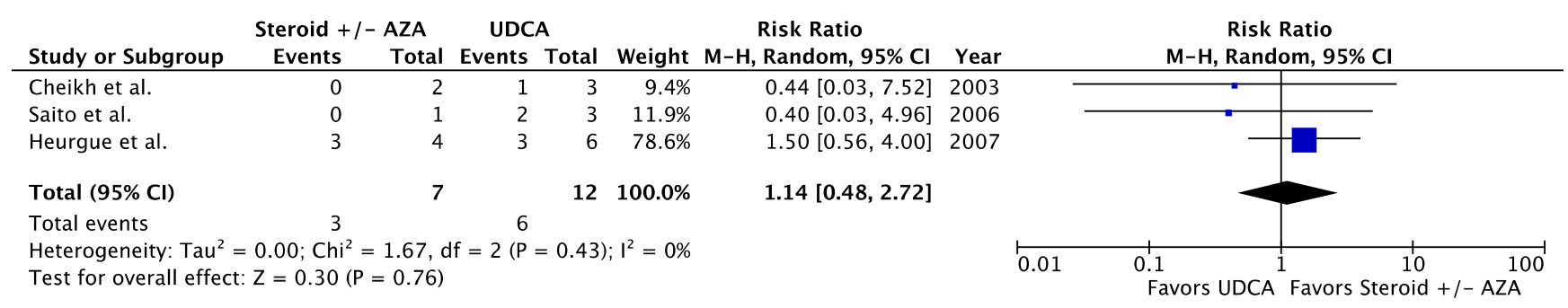

Figure 5. Biochemical improvement in AIH-PBC patients treated with immunosuppression vs. UDCA. UDCA = ursodeoxycholic acid, steroid = corticosteroids, AZA $=$ azathioprine.

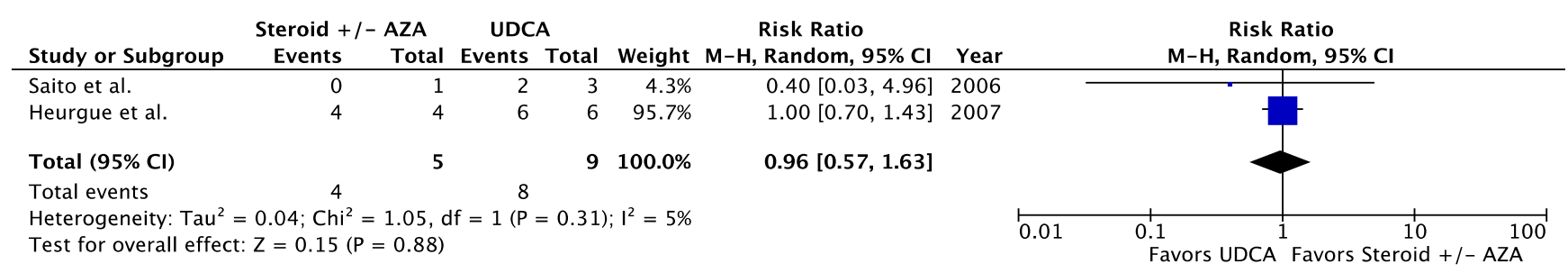

Figure 6. Transplant-free survival in AIH-PBC patients treated with immunosuppression vs. UDCA. UDCA = ursodeoxycholic acid, steroid = corticosteroids, AZA $=$ azathioprine.

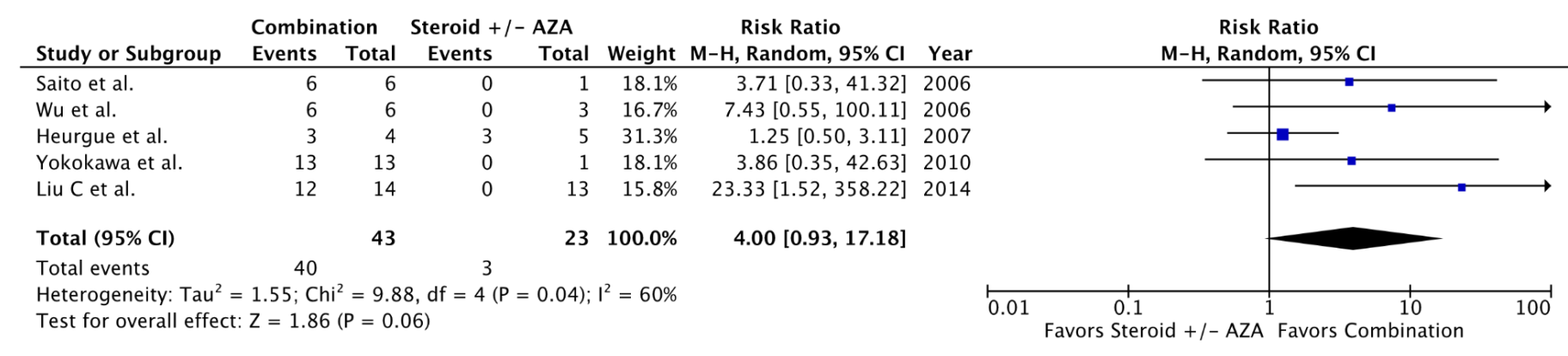

Figure 7. Biochemical improvement in AIH-PBC patients treated with combination therapy vs. immunosuppression. Steroid $=$ corticosteroids, $\mathrm{AZA}=$ azathioprine, Combination $=$ UDCA + corticosteroids \pm AZA . 


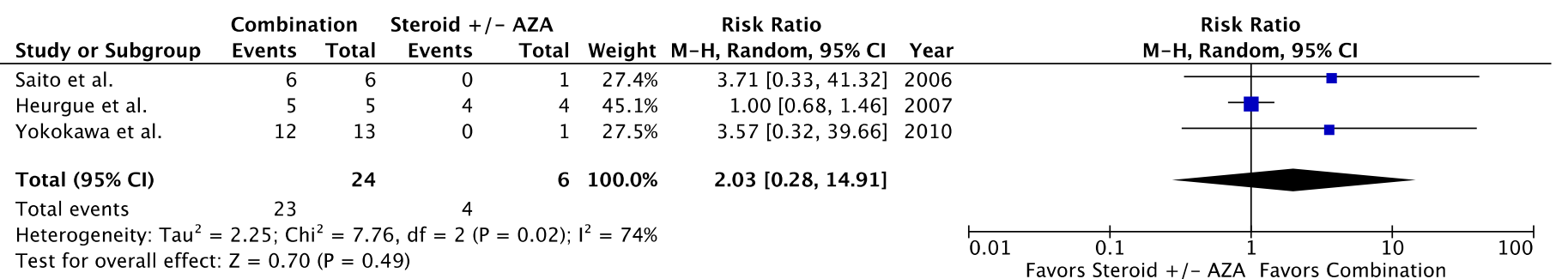

Figure 8. Transplant-free survival in AIH-PBC patients treated with combination therapy vs. immunosuppression. Steroid = corticosteroids, AZA $=$ azathioprine, Combination $=$ UDCA + corticosteroids \pm AZA . 
Table 3. Results of studies included in systematic review but excluded from meta-analysis.

\begin{tabular}{|c|c|c|c|c|c|c|c|}
\hline Study & Treatments Compared & $\begin{array}{c}\text { Symptom } \\
\text { Improvement }\end{array}$ & $\begin{array}{c}\text { Biochemical } \\
\text { Improvement }\end{array}$ & $\begin{array}{l}\text { Improved } \\
\text { Histologic } \\
\text { Activity }\end{array}$ & $\begin{array}{l}\text { Fibrosis Non- } \\
\text { Progression }\end{array}$ & $\begin{array}{c}\text { Transplant- } \\
\text { Free Survival }\end{array}$ & $\begin{array}{l}\text { Reason for Exclusion } \\
\text { from Meta-Analysis }\end{array}$ \\
\hline \multicolumn{8}{|l|}{$A I H-P B C$} \\
\hline \multirow{2}{*}{ Joshi 2002 [8] } & UDCA & - & - & $3 / 9$ & - & $-^{*}$ & \multirow{2}{*}{$\begin{array}{l}\text { Only study comparing } \\
\text { UDCA to placebo }\end{array}$} \\
\hline & Placebo & - & - & $0 / 2$ & - & $-^{*}$ & \\
\hline \multirow{2}{*}{ Lindgren 2009 [22] } & UDCA & - & $3 / 18$ & - & - & - & \multirow{2}{*}{$\begin{array}{l}\text { Overlapping treatment } \\
\text { groups (see Table 1) }\end{array}$} \\
\hline & Corticosteroids & - & $5 / 15$ & - & - & - & \\
\hline \multirow{2}{*}{ Liu 2014 [69] } & UDCA & - & $0 / 6$ & - & - & - & \multirow{2}{*}{$\begin{array}{l}\text { No endpoints reached in } \\
\text { either treatment group }\end{array}$} \\
\hline & UDCA + prednisone \pm & - & $0 / 1$ & - & - & - & \\
\hline \multirow{2}{*}{ Serghini 2012 [72] } & UDCA + corticosteroids + AZA & - & $0 / 4$ & - & - & - & \multirow{2}{*}{$\begin{array}{l}\text { No endpoints reached in } \\
\text { either treatment group }\end{array}$} \\
\hline & Corticosteroids + AZA & - & $0 / 1$ & - & - & - & \\
\hline \multicolumn{8}{|l|}{ AIH-PSC } \\
\hline \multirow[b]{2}{*}{ Luth 2009 [35] } & Corticosteroids \pm & - & $9 / 10$ & - & - & - & \multirow{2}{*}{$\begin{array}{c}\text { No comparator study for } \\
\text { biochemical improvement } \\
\text { (see below) }\end{array}$} \\
\hline & UDCA + corticosteroids \pm AZA & - & $6 / 6$ & - & - & - & \\
\hline \multirow{2}{*}{ McNair 1998 [41] } & Prednisolone + AZA & $2 / 3$ & $0 / 3$ & $2 / 3$ & $2 / 3$ & $2 / 3$ & \multirow{2}{*}{$\begin{array}{l}\text { No biochemical endpoints } \\
\text { reached in either group }\end{array}$} \\
\hline & $\mathrm{UDCA}+$ prednisolone $+\mathrm{AZA}$ & $2 / 2$ & $0 / 2$ & $0 / 1^{+}$ & $1 / 1^{\dagger}$ & $2 / 2$ & \\
\hline \multicolumn{8}{|l|}{ AIC } \\
\hline \multirow{3}{*}{ Campos 2017 [23] } & Prednisolone, then UDCA, cholestyramine, rifampicin, & - & $1 / 1$ & - & - & $1 / 1$ & \multirow{3}{*}{$\begin{array}{c}\text { No comparator studies } \\
\text { with similar } \\
\text { treatment groups }\end{array}$} \\
\hline & $\begin{array}{l}\text { naitrexone, sertramne, nyarodyzine, amitriptyme, } \\
\text { phototherapy, molecular adsorbent recirculating }\end{array}$ & & & & & & \\
\hline & system, prednisolone, budesonide, AZA, MMF & $0 / 1$ & $0 / 1$ & - & - & $1 / 1$ & \\
\hline \multicolumn{8}{|l|}{$A S C$} \\
\hline \multirow{2}{*}{ Gregorio 2001 [33] } & UDCA & - & - & $3 / 3$ & - & - & \multirow{2}{*}{$\begin{array}{l}\text { No endpoint is reported } \\
\text { for both treatment groups }\end{array}$} \\
\hline & Prednisolone \pm UDCA \pm AZA & - & $20 / 23$ & - & - & - & \\
\hline
\end{tabular}

Transplant-free survival is expressed as the raw fraction of study participants who did not experience death or liver transplant by the end of follow-up. Fibrosis and histologic activity were assessed using the METAVIR scoring system [83] except where otherwise noted. In treatment regimens comprising "corticosteroids", the authors did not specify which corticosteroid(s) were used. The treatment comparison in Joshi et al. was reported as statistically non-significant; statistical testing for the other studies was not reported. * Transplant-free survival was omitted from this analysis given cross-over of four patients from placebo to the UDCA group after $\sim 2$ years. ${ }^{+}$Liver biopsy results were reported for only one of the two patients in this treatment group. AIH: Autoimmune hepatitis, PBC: Primary biliary cholangitis, PSC: Primary sclerosing cholangitis, AIC: Autoimmune cholangitis, ASC: Autoimmune sclerosing cholangitis, UDCA: Ursodeoxycholic acid, MMF: Mycophenolate mofetil. 


\subsubsection{AIH-PSC}

Although neither study was eligible for meta-analysis, Luth et al. and McNair et al. compared combination therapy to corticosteroids \pm AZA for the treatment of AIH-PSC. Luth et al. followed 16 patients for a median of 144 months, and observed biochemical improvement in all six patients treated with combination therapy and nine of 10 patients treated with corticosteroids \pm AZA. They did not report other treatment outcomes [35]. McNair et al. followed five patients for a median of 84 months, and observed symptomatic improvement in two of two patients treated with combination therapy and two of three patients treated with prednisolone + AZA. No biochemical improvement was observed in either treatment group. Histologic activity was improved in two of the three patients treated with prednisolone + AZA but unchanged in the one patient treated with combination therapy who underwent a second liver biopsy. No progression in liver fibrosis was seen in this patient, nor in two out of the three patients treated with prednisolone + AZA. Aside from one patient in the prednisolone + AZA group who died from liver failure after six years of follow-up, all patients survived without need for liver transplantation (Table 3) [41].

\subsubsection{AIC}

When two studies comprised of 20 patients with AIC were meta-analyzed to compare corticosteroids \pm AZA to UDCA alone, no difference was seen in the rate of biochemical improvement $\left(\mathrm{RR}=0.59,95 \% \mathrm{CI} 0.16-2.17, p=0.43, \mathrm{I}^{2}=0 \%\right.$ ) (Figure 9). Excluded from the meta-analysis given its unique treatment comparison, Campos et al. reported biochemical improvement in one AIC patient treated with UDCA $(750 \mathrm{mg} / \mathrm{d})$ and prednisolone, while another patient failed to realize symptomatic or biochemical improvement despite a complex regimen including several rescue therapies (Table 3) [23].

\subsubsection{ASC}

When two studies comprised of 25 patients with ASC were meta-analyzed to compare combination therapy to UDCA alone, no differences in biochemical improvement $(\mathrm{RR}=1.07,95 \% \mathrm{CI} 0.66-1.74$, $\left.p=0.78, \mathrm{I}^{2}=0 \%\right)$ or transplant-free survival $\left(\mathrm{RR}=1.00,95 \% \mathrm{CI} 0.69-1.45, p=1.00, \mathrm{I}^{2}=0 \%\right)$ were identified between the two treatment groups (Figures 10 and 11). Excluded from the meta-analysis because of incomplete reporting of treatment outcomes, Gregorio et al. observed biochemical improvement in 20 of 23 AIC patients treated with combination therapy and improved histologic activity in all three patients treated with UDCA alone (Table 3) [33].

\subsection{Quality of Evidence}

The treatment effect estimates derived from each of our meta-analyses constitute very low quality evidence, given their reliance solely on observational studies and the failure of those individual studies to control for potentially confounding variables (i.e., poor study design by GRADE criteria) [63]. Another common limitation was imprecision due to suboptimal information size, which affected the following meta-analyses: UDCA vs. corticosteroids \pm AZA for biochemical improvement in AIC, UDCA vs. combination therapy for biochemical improvement in ASC, UDCA vs. combination therapy for symptomatic improvement in AIH-PBC, UDCA vs. corticosteroids \pm AZA for biochemical improvement in AIH-PBC, UDCA vs. combination therapy for fibrosis non-progression in AIH-PBC, UDCA vs. corticosteroids \pm AZA for transplant-free survival in AIH-PBC, and corticosteroids \pm AZA vs. combination therapy for transplant-free survival in AIH-PBC. Furthermore, the meta-analyses of UDCA vs. combination therapy for biochemical improvement and transplant-free survival in AIH-PBC may have suffered from publication bias (Figure 12) and the studies meta-analyzed in the comparison of UDCA to combination therapy for transplant-free survival in ASC had follow-up times that were too short for deaths or liver transplantations to occur. 


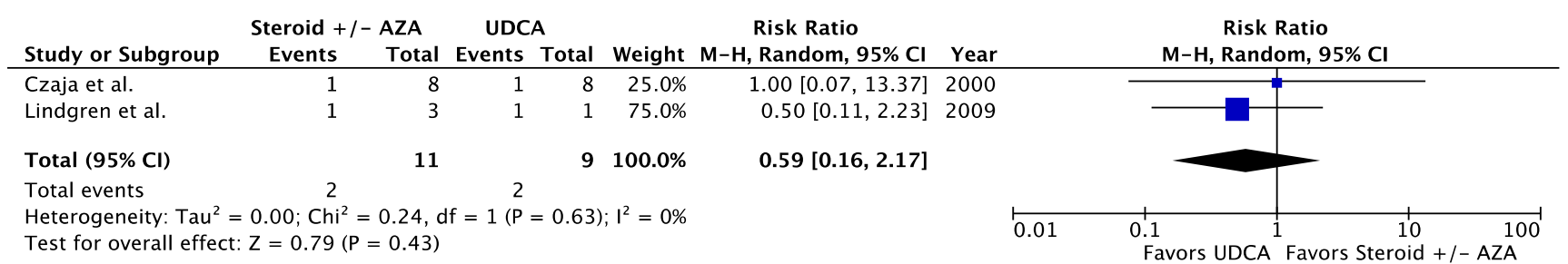

Figure 9. Biochemical improvement in AIC patients treated with immunosuppression vs. UDCA. Steroid $=$ corticosteroids, $\mathrm{AZA}=$ azathioprine, and UDCA $=$ ursodeoxycholic acid.

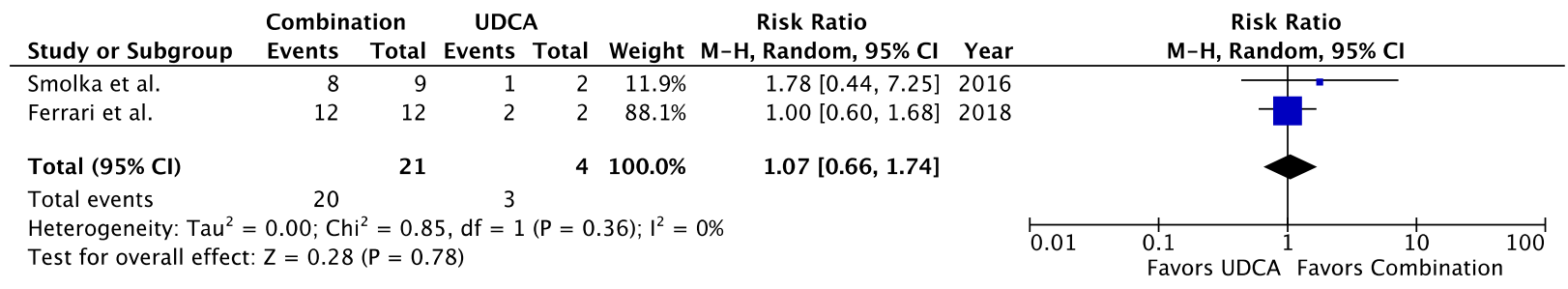

Figure 10. Biochemical improvement in ASC patients treated with combination therapy vs. UDCA. UDCA $=$ ursodeoxycholic acid and Combination $=$ UDCA + prednisone $\pm \mathrm{AZA}$

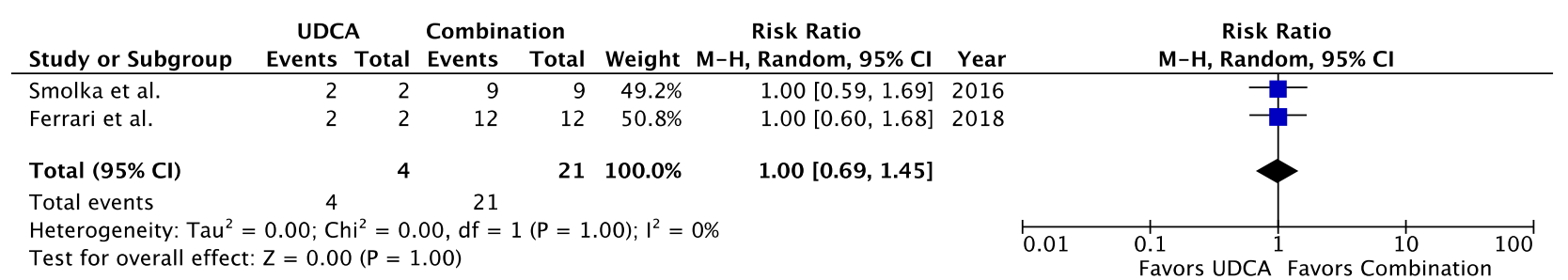

Figure 11. Transplant-free survival in ASC patients treated with combination therapy vs. UDCA. UDCA $=$ ursodeoxycholic acid and Combination $=$ UDCA + prednisone \pm AZA. 


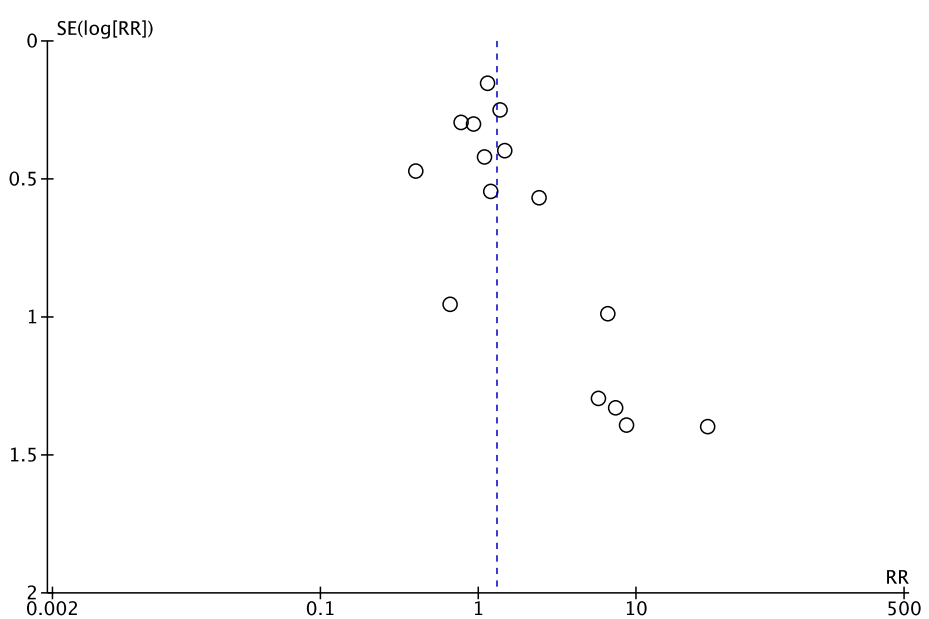

(a)

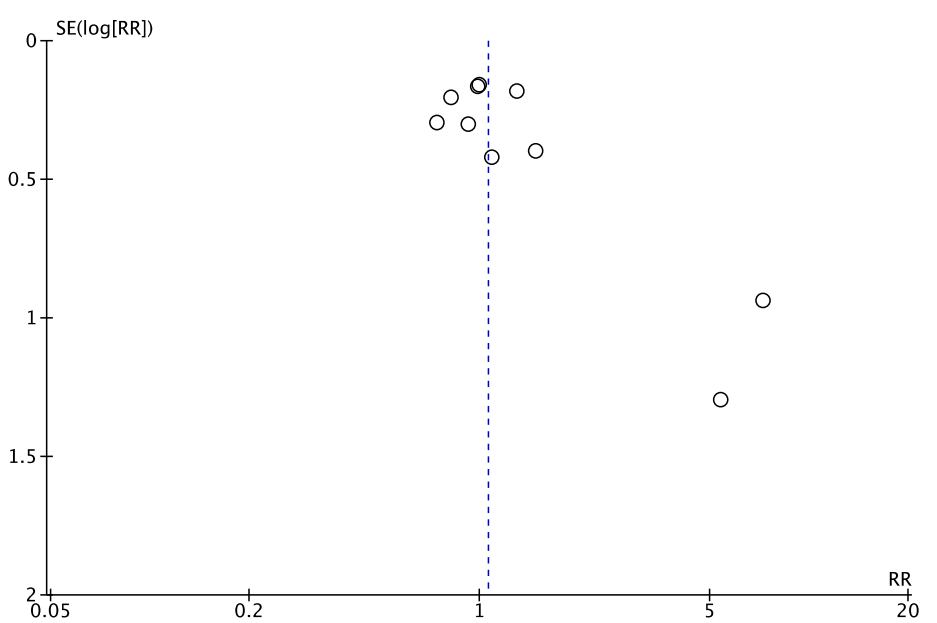

(b)

Figure 12. Funnel plots of AIH-PBC studies meta-analyzed to compare (a) biochemical improvement and (b) transplant-free survival among patients treated with UDCA vs. combination therapy. UDCA $=$ ursodeoxycholic acid and combination therapy $=$ UDCA + [corticosteroids and/or antimetabolites]. 


\section{Discussion}

Overlap syndromes in autoimmune liver disease are morbid conditions whose rigorous study has been made difficult by their rarity in the general population. Despite numerous small studies over the past few decades, uncertainty remains regarding optimal treatment strategies for these syndromes. Through conducting the above meta-analyses and systematic review of the primary literature, we therefore endeavored to collate all comparative data on first-line therapies for AIH-PBC, AIH-PSC, PBC-PSC, AIH-PBC-PSC, AIC, and ASC, and to determine which therapies, if any, were more effective than others. Ultimately, we demonstrated that combination therapy with UDCA and immunosuppression may be superior both to UDCA alone and to corticosteroids \pm AZA for the treatment of AIH-PBC with respect to biochemical improvement and transplant-free survival. The studies of non-AIH-PBC overlap syndromes were either ineligible for our systematic review (PBC-PSC, and AIH-PBC-PSC) or meta-analysis (AIH-PSC) or showed no treatment effect when meta-analyzed (AIC and ASC), highlighting the importance of further primary research in this area.

The most studied treatments for AIH-PBC have been done with UDCA alone, or a combination of UDCA with corticosteroids and/or other immunosuppressive agents (e.g., MMF, AZA). Interest in comparing these two treatment options stems at least in part from a desire to avoid treating patients unnecessarily with immunosuppressive medications that may cause harmful side effects [58]. Our initial meta-analysis of this treatment comparison, pooling 349 patients across 15 studies with AIH-PBC, did not show a difference in biochemical improvement between the two treatment groups.

While we believe this null result should prompt additional studies, it does not preclude the possibility that combination therapy may in fact be superior to UDCA alone for patients with AIH-PBC, or at least a subset thereof. In a cohort of 88 patients with AIH-PBC, Ozaslan et al. showed that the presence of severe interface hepatitis on liver biopsy was an independent predictor of failure to achieve biochemical remission on UDCA monotherapy but not on combination therapy [10]. Hence, adding immunosuppressive therapy to UDCA may be of significant benefit in AIH-PBC with severe interface hepatitis but of little or no benefit in patients with lesser degrees of interface hepatitis, which is a concept highlighted by the EASL 2017 PBC guidelines [59]. Since most studies comparing UDCA to combination therapy in AIH-PBC have been comprised of patients with a wide range of histologic severity and have not stratified treatment outcomes by degree of baseline interface hepatitis, a subgroup analysis to assess this hypothesis further was not feasible in our meta-analysis, and a treatment effect could therefore have been masked.

The fact that our sensitivity analysis including only those studies which defined AIH-PBC using the Paris criteria did not reveal a difference in biochemical improvement between treatment groups suggests that if the null finding of our meta-analysis represented type II error, the error was not driven by excessive heterogeneity in the definition of AIH-PBC. Several studies of AIH-PBC that reported biochemical improvement as a clinical outcome either failed to define it altogether [67,68] or defined it only in terms of hepatocellular markers [6,7,22,25,66,69-71,75]. Intuitively, for an overlap syndrome driven by both hepatocellular inflammation and cholestasis, it did not seem as though improved hepatocellular markers alone would make a very reliable surrogate endpoint for longer-term outcomes (e.g., transplant-free survival). To maximize the uniformity and clinical meaningfulness of biochemical improvement as an outcome for AIH-PBC patients, we therefore performed a second sensitivity analysis including only those studies which defined biochemical improvement in terms of both hepatocellular and cholestatic markers [8,10,11,57,65,73,74]. The latter comparison included 202 patients across seven studies, and revealed a non-significant trend toward the superiority of combination therapy over UDCA ( $R R=1.34,95 \%$ CI 0.93-1.93). This finding is considered exploratory, since the sensitivity analysis was not pre-specified.

Non-progression of liver fibrosis was reported in relatively few patients with AIH-PBC. Therefore, we were able to meta-analyze only two studies with a total of 46 patients, comparing UDCA to combination therapy [6,10]. With consequently limited statistical power, our meta-analysis did not detect a difference in non-progression of liver fibrosis between treatment groups. However, it is 
worthwhile noting that one of the two studies, the only one without cross-over of patients between treatment groups, found a significant benefit of combination therapy relative to UDCA $(R R=2.20$, 95\% CI 1.02-4.74) [6]. The null result of the second study may have been confounded by the cross-over of nine patients from the UDCA group to the combination therapy group, most of whom had no intervening liver biopsy [10]. Therefore, progression of liver fibrosis may have occurred in several of these individuals while on UDCA monotherapy and been subsequently misattributed to combination therapy after they switched between treatment groups.

While we found no difference in transplant-free survival between UDCA and combination therapy in a meta-analysis of 170 AIH-PBC patients across 10 studies, our confidence in this result was limited by the relatively small number of deaths and liver transplants that occurred. Four of the individual studies, for example, tallied $\leq 1$ death or liver transplant each $[6,8,57,71]$. This phenomenon may have resulted from a combination of having an inadequate duration of follow-up, inadequate study sizes, or disproportionate enrollment of patients early in their disease course with favorable prognoses. When the meta-analysis was restricted to the two studies with a follow-up period $>90$ months [74,75], combination therapy was associated with a significantly higher transplant-free survival than UDCA alone ( $R R=6.50,95 \%$ CI 1.47-28.83). This finding should be regarded as hypothesis-generating, given that the sensitivity analysis above was not pre-specified. Furthermore, because both studies transpired at Japanese medical centers, the generalizability of this result may be hindered by ethnic homogeneity.

Few studies of overlap syndromes reported patients' symptoms and how they changed with treatment and indeed we were able to meta-analyze only two studies (including 40 patients with AIH-PBC), comparing UDCA to combination therapy with respect to symptomatic improvement $[25,66]$. Reported symptoms of AIH-PBC included pruritis [25], fatigue [25,66], jaundice [25,66], weight loss [25,66], arthritis/arthralgia [25,66], myalgia [25], lower limb swelling [66], abdominal pain [66], and nausea [66]. The null result of this meta-analysis could suggest an extra-hepatic etiology of some of these symptoms and while combination therapy may enhance biochemical improvement and transplant-free survival compared to UDCA, both liver-directed therapies may be equivalent (and perhaps no better than placebo) in modifying symptoms of AIH-PBC if the latter originated outside the liver. A similar hypothesis was proposed to explain UDCA's failure to alleviate PBC-related fatigue in a meta-analysis of several randomized trials [84]. Alternatively, it is plausible that combination therapy is more effective than UDCA alone at alleviating a particular symptom of AIH-PBC, but that this effect was obscured by the design of the two meta-analyzed cohort studies, both of which considered a group of several symptoms together rather than individually $[25,66]$.

As UDCA is generally well tolerated (and thus, there is little perceived disadvantage to adding it empirically to an immunosuppressive regimen), few studies have examined immunosuppressive therapy alone for AIH-PBC. While some case series suggest that corticosteroids \pm AZA may suffice to induce remission [3,14], all five cohort studies comparing immunosuppression alone to combination therapy $[8,68,71,73,74]$ showed at least a trend toward more biochemical improvement with combination therapy, which approached statistical significance upon meta-analysis $(R R=4.00,95 \%$ CI 0.93-17.18). With only three studies comprising 30 patients who experienced cumulatively only three deaths or liver transplants [8,71,74], our meta-analysis comparing combination therapy to corticosteroids \pm AZA with respect to transplant-free survival detected no difference between treatment groups but should be interpreted cautiously in the context of limited statistical power. Similarly, a paucity of studies comparing UDCA to corticosteroids \pm AZA in AIH-PBC with respect to biochemical improvement or transplant-free survival precludes a definitive interpretation of our corresponding meta-analyses, both of which failed to demonstrate the superiority of either treatment regimen.

Our decision to censor patients who crossed between treatment groups (in an effort to maximize statistical uniformity) may have contributed to an underestimate of the true impact of combination therapy (compared to UDCA or immunosuppression alone) on clinical outcomes in AIH-PBC. For example, $\mathrm{Wu}$ et al. observed biochemical improvement in zero out of three AIH-PBC patients treated with UDCA, zero out of three treated with prednisone, and six out of six treated with UDCA + 
prednisone as first-line therapies. This apparent superiority of combination therapy is accentuated if one considers that all six patients from this study who failed monotherapy subsequently responded to UDCA + prednisone as second-line therapy [73]. In this or other scenarios where patients switch from mono- to combination therapy after failing the former, confounding factors (e.g., more advanced liver disease), if present, seem more likely to underestimate than to overestimate the effectiveness of combination therapy.

No difference in biochemical improvement was seen between UDCA and corticosteroids \pm AZA in a meta-analysis of two studies comprising 20 patients with AIC. However, combination therapy may be a more effective option for these patients, as suggested by the symptomatic and biochemical remission of nine out of 10 patients with "AIH-AIC" treated with UDCA + corticosteroids \pm AZA in a case series conducted by Ozaslan et al. [25]. Furthermore, as noted elsewhere, obtaining a deeper pathophysiologic understanding and a more specific diagnostic definition of AIC may facilitate the optimization of treatment strategies [1].

The few cohort studies of ASC meeting inclusion criteria for our systematic review suggest a fairly positive prognosis for these patients, whether they are treated with UDCA or combination therapy $[33,43,44]$. In the two meta-analyzed studies, 20 of 21 patients on combination therapy and three of four patients on UDCA experienced biochemical improvement, and all 25 patients survived without needing a liver transplant $[43,44]$. This contrasts with the $65 \%$ transplant-free survival reported by Rodrigues et al. in a case series of 28 ASC patients treated with combination therapy however, the prevalence of cirrhosis on initial presentation was considerably higher $(77 \%)$ in the latter study, compared to $\sim 16 \%$ and $0 \%$ in Ferrari and Smolka et al., respectively [36,43,44]. To discern the relative efficacy of different treatment regimens for ASC, more studies are required, ideally with larger patient cohorts and/or longer follow-up periods.

It is difficult to draw meaningful conclusions from the two studies of AIH-PSC in our systematic review, which compared corticosteroids \pm AZA to combination therapy and were not eligible for meta-analysis. Luth et al. observed biochemical improvement in almost all patients regardless of treatment group, but did not report histologic outcomes or transplant-free survival, and McNair et al. reported multiple clinical outcomes in only five patients $[35,41]$. We could not compare the effectiveness of different treatment strategies for PBC-PSC or AIH-PBC-PSC, given that our systematic literature search yielded only case series and case reports of these syndromes.

The most important limitation of our systematic review and meta-analysis is its considerable risk of bias, resulting from the lack of treatment randomization or other techniques to minimize confounding in its constituent studies. This highlights the need for multi-center randomized trials and larger observational studies that employ matched patient selection or multivariable modeling to control for confounding factors (e.g., age, comorbidities, and degree of liver fibrosis or histologic activity). Furthermore, our analysis may have forfeited a degree of nuance by not including clinical outcomes of intermediate severity reported in some studies (e.g., decompensated cirrhosis) [11,43]. Lastly, our systematic review examined only first-line pharmacotherapies. Given increasing experimentation with MMF and calcineurin inhibitors as rescue therapies for overlap syndromes [8,10,15-17,45], a subsequent review of second-line therapies and their comparative effectiveness may be warranted.

\section{Conclusions}

UDCA, immunosuppression, or a combination of both have been used as first line therapies for overlap syndromes in autoimmune liver disease, but evidence supporting one regimen over the others is sparse. Our systematic review and meta-analysis showed no clear differences in clinical outcomes between these treatment regimens in any of the examined overlap syndromes, although the quality of evidence was very low. While awaiting more definitive studies, providers should continue to rely on professional society guidelines and expert opinion in the treatment of these rare diseases. 
Author Contributions: Conceptualization, B.L.F., C.J.D., and A.B.; formal analysis, B.L.F. and C.J.D.; investigation, B.L.F.; writing-original draft preparation, B.L.F; writing-review and editing, A.B. and V.P.; supervision, C.J.D. and A.B. All authors have read and agreed to the published version of the manuscript.

Funding: This research received no external funding.

Acknowledgments: Guidance regarding literature search strategies was provided by Harvard Medical School Research and Education Librarian Paul Bain, PhD, MLIS.

Conflicts of Interest: The authors declare no conflict of interest. 


\section{Appendix A}

Table A1. Search strategies.

$\begin{array}{lll}\text { Database } & \text { Time Span } & \text { Filter }\end{array}$

$\begin{array}{lll}\text { Database } & \text { Time Span } & \text { Filter }\end{array}$

$\begin{array}{lll}\text { Database } & \text { Time Span } & \text { Filter }\end{array}$

PubMed Searched on 30

September 2019

None

restricted to articles,

articles in press, or

September 2019

conference papers

Cochrane

Searched on 30

September 2019

Study type restricted

to clinical trials

Restricted to articles,

clinical trials, data sets,

Web of

Searched on 30

Science data studies, "early

access", unspecified

or "other"
Search Strategy

(overlap syndrome[Title/Abstract] AND ("hepatitis, autoimmune”[MeSH Terms] OR autoimmune hepatitis[Title/Abstract] OR autoimmune liver disease[Title/Abstract] OR "liver cirrhosis, biliary"[MeSH Terms] OR primary biliary

cirrhosis[Title/Abstract] OR primary biliary cholangitis[Title/Abstract] OR "cholangitis, sclerosing"[MeSH Terms] OR

primary sclerosing cholangitis[Title/Abstract])) OR ((“hepatitis, autoimmune”[MeSH Terms] OR autoimmune

hepatitis[Title/Abstract]) AND ("liver cirrhosis, biliary"[MeSH Terms] OR primary biliary cirrhosis[Title/Abstract] OR primary biliary cholangitis[Title/Abstract])) OR (("hepatitis, autoimmune”[MeSH Terms] OR autoimmune

hepatitis[Title/Abstract]) AND ("cholangitis, sclerosing"[MeSH Terms] OR primary sclerosing cholangitis[Title/Abstract])) OR (("liver cirrhosis, biliary"[MeSH Terms] OR primary biliary cirrhosis[Title/Abstract] OR primary biliary cholangitis[Title/Abstract]) AND ("cholangitis, sclerosing”[MeSH Terms] OR primary sclerosing cholangitis[Title/Abstract])) OR Autoimmune cholangitis[Title/Abstract] OR Autoimmune sclerosing cholangitis[Title/Abstract]

(('overlap syndrome'/exp OR 'overlap syndrome':ab,ti) AND ('autoimmune hepatitis'/exp OR 'autoimmune hepatitis':ab,ti OR 'autoimmune liver disease'/exp OR 'autoimmune liver disease':ab,ti OR 'primary biliary cirrhosis'/exp OR 'primary biliary cirrhosis':ab,ti OR 'primary biliary cholangitis':ab,ti OR 'primary sclerosing cholangitis'/exp OR 'primary sclerosing cholangitis':ab,ti)) OR (('autoimmune hepatitis'/exp OR 'autoimmune hepatitis':ab,ti) AND ('primary biliary cirrhosis'/exp OR 'primary biliary cirrhosis':ab, ti OR 'primary biliary cholangitis':ab,ti)) OR (('autoimmune hepatitis'/exp OR 'autoimmune hepatitis':ab,ti) AND ('primary sclerosing cholangitis'/exp OR 'primary sclerosing cholangitis':ab,ti)) OR (('primary biliary cirrhosis'/exp OR 'primary biliary cirrhosis':ab,ti OR 'primary biliary cholangitis':ab,ti) AND ('primary sclerosing cholangitis'/exp OR 'primary sclerosing cholangitis':ab,ti)) OR 'Autoimmune cholangitis'/exp OR 'autoimmune cholangitis':ab,ti OR 'Autoimmune sclerosing cholangitis'/exp OR 'autoimmune sclerosing cholangitis':ab,ti

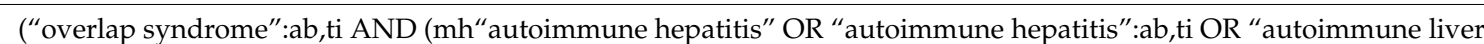
disease":ab,ti OR mh"primary biliary cirrhosis" OR "primary biliary cirrhosis":ab,ti OR "primary biliary cholangitis":ab,ti OR mh"primary sclerosing cholangitis" OR "primary sclerosing cholangitis":ab,ti)) OR ((mh"autoimmune hepatitis" OR "autoimmune hepatitis":ab,ti) AND (mh"primary biliary cirrhosis" OR "primary biliary cirrhosis":ab,ti OR "primary biliary cholangitis":ab,ti)) OR ((mh"autoimmune hepatitis" OR "autoimmune hepatitis":ab,ti) AND (mh"primary sclerosing cholangitis" OR "primary sclerosing cholangitis":ab,ti)) OR ((mh"primary biliary cirrhosis" OR "primary biliary

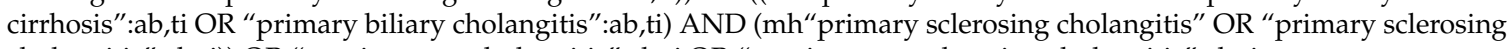
cholangitis":ab,ti)) OR “autoimmune cholangitis":ab,ti OR "autoimmune sclerosing cholangitis":ab,ti

(TS = ("overlap syndrome") AND (TS = ("autoimmune hepatitis") OR TS = ("autoimmune liver disease")

OR TS $=($ "primary biliary cirrhosis") OR TS $=$ ("primary biliary cholangitis")

OR TS = ("primary sclerosing cholangitis")) $)$ OR $($ TS = ("autoimmune hepatitis") AND $($ TS = ("primary biliary cirrhosis") OR TS = ("primary biliary cholangitis")) $)$ OR (TS = ("autoimmune hepatitis") AND TS = ("primary sclerosing cholangitis")) OR $(($ TS = ("primary biliary cirrhosis") OR TS = ("primary biliary cholangitis") $)$ AND TS = ("primary sclerosing cholangitis")) OR TS = ("Autoimmune cholangitis") OR TS = ("Autoimmune sclerosing cholangitis") 


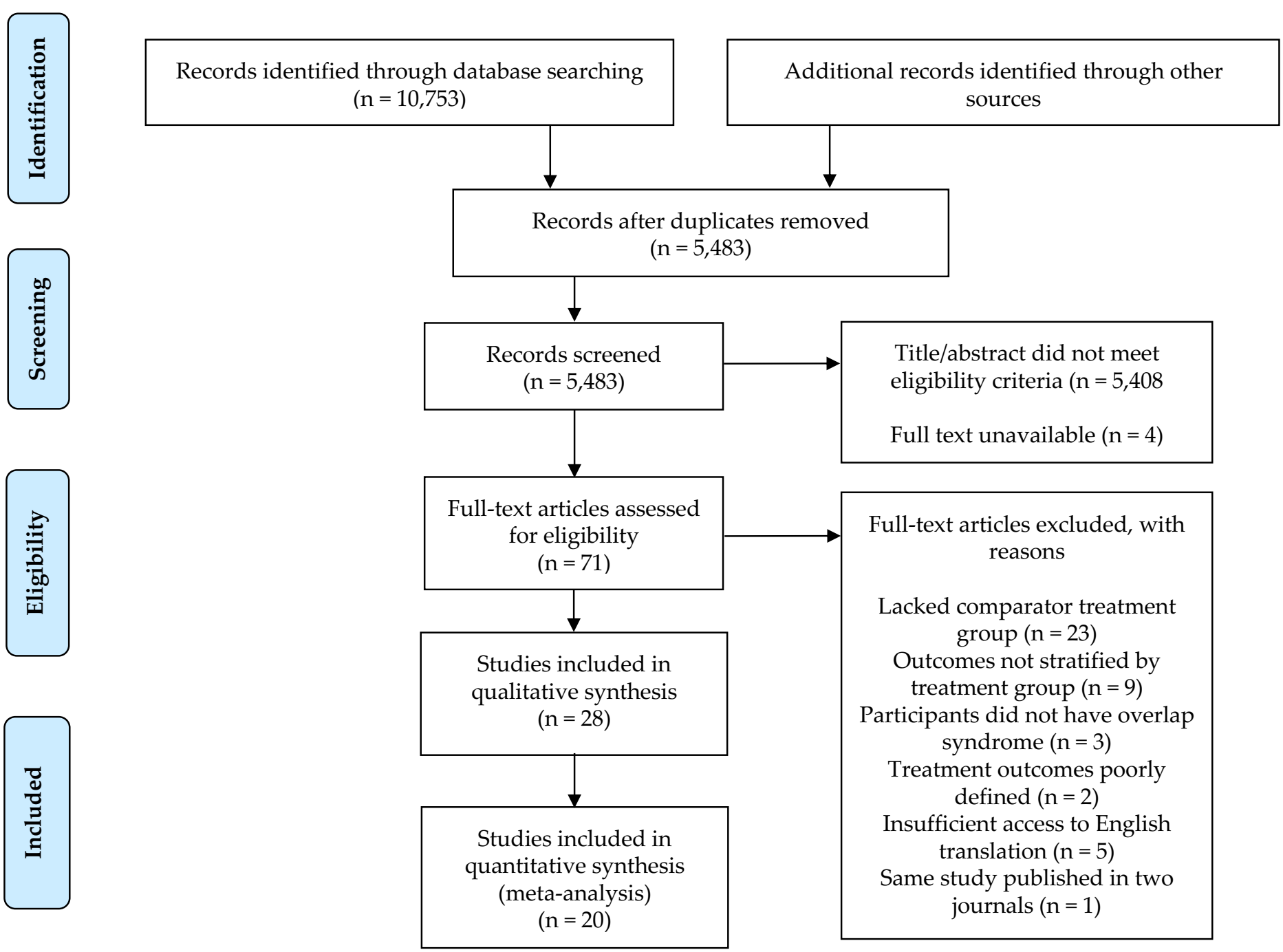

Figure A1. Study selection flow diagram. 


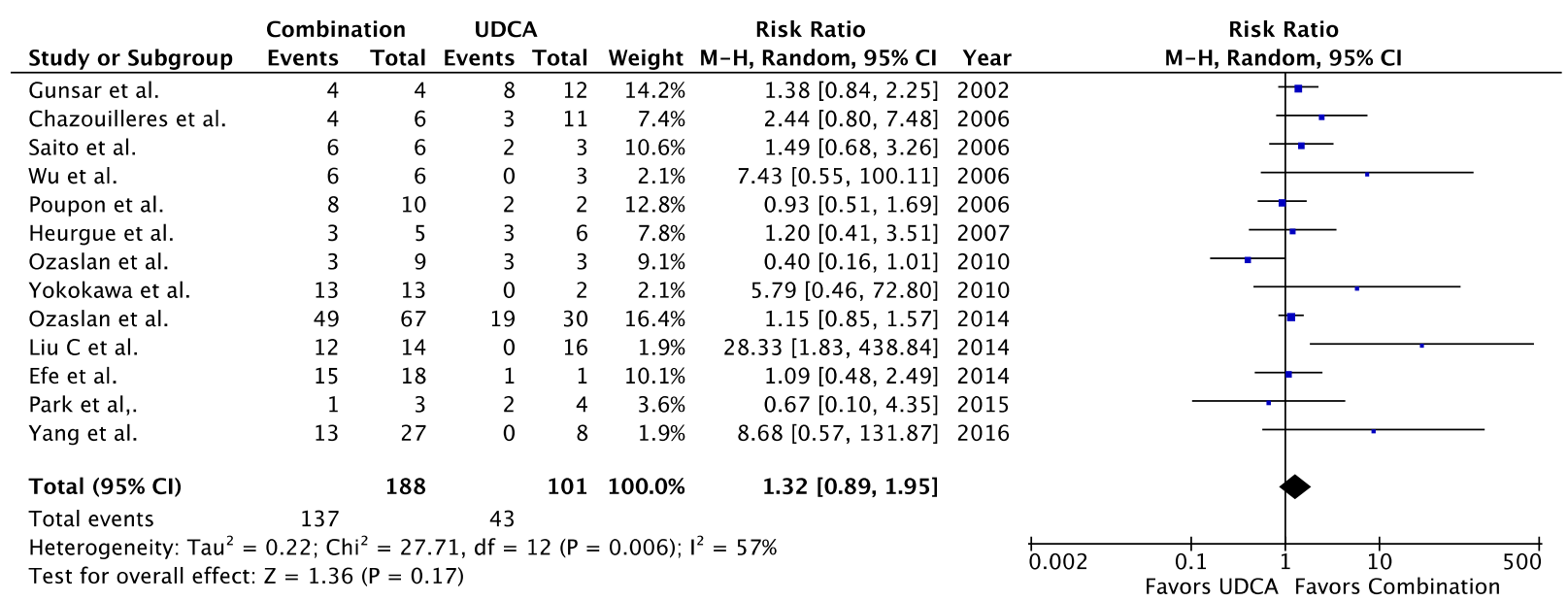

Figure A2. Biochemical improvement in AIH-PBC patients treated with combination therapy vs. UDCA alone, including only those studies in which combination therapy consisted of UDCA + corticosteroids \pm AZA (i.e., no patients were treated with MMF, or with a combination of UDCA and antimetabolite therapy without corticosteroids). UDCA = ursodeoxycholic acid, $A Z A=$ azathioprine, $M M F=$ mycophenolate mofetil.

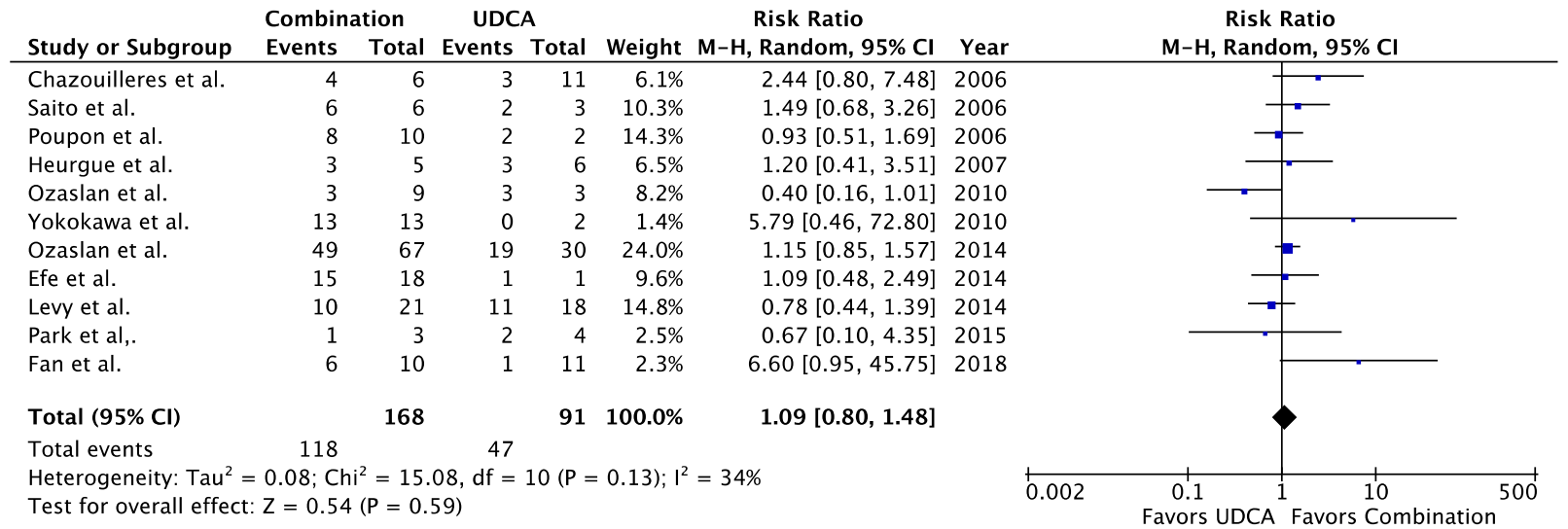

Figure A3. Biochemical improvement in AIH-PBC patients treated with combination therapy vs. UDCA alone, including only those studies in which AIH-PBC was defined by the Paris criteria. UDCA = ursodeoxycholic acid, Combination = UDCA + [corticosteroids and/or antimetabolites]. 


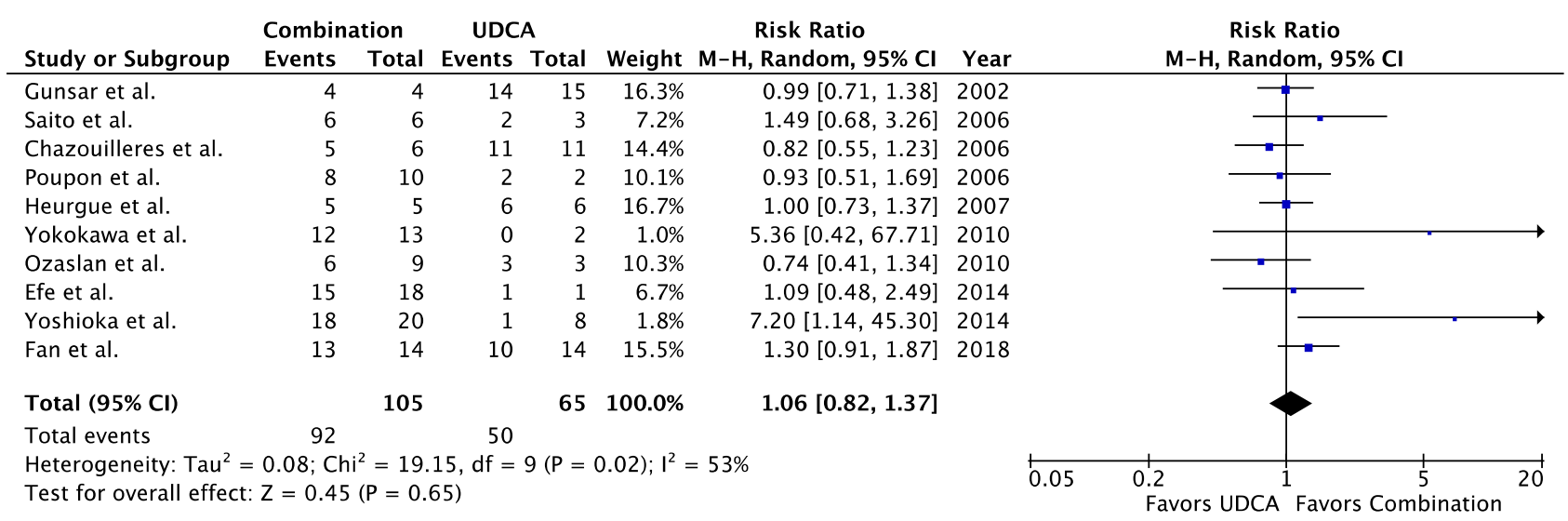

Figure A4. Biochemical improvement in AIH-PBC patients treated with combination therapy vs. UDCA alone, including only those studies in which biochemical improvement was defined by both hepatocellular and cholestatic markers. UDCA $=$ ursodeoxycholic acid, Combination $=$ UDCA $+[$ corticosteroids and/or antimetabolites].

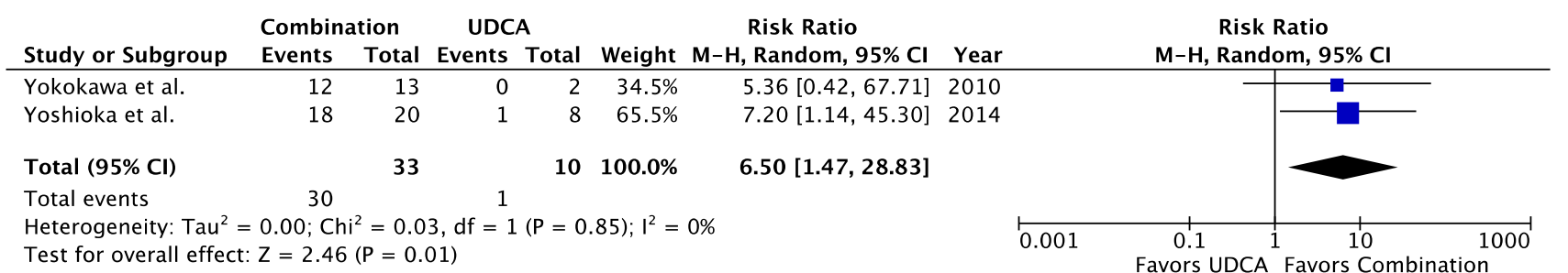

Figure A5. Transplant-free survival in AIH-PBC patients treated with combination therapy vs. UDCA alone, including only those studies in which mean or median follow-up time exceeded 90 months. UDCA = ursodeoxycholic acid, Combination $=$ UDCA + corticosteroids \pm AZA. 


\section{References}

1. Czaja, A.J.; Carpenter, H.A. Autoimmune Hepatitis Overlap Syndromes and Liver Pathology. Gastroenterol. Clin. N. Am. 2017, 46, 345-364. [CrossRef] [PubMed]

2. Bonder, A.; Retana, A.; Winston, D.M.; Leung, J.; Kaplan, M. Prevalence of Primary Biliary CirrhosisAutoimmune Hepatitis Overlap Syndrome. Clin. Gastroenterol. Hepatol. 2011, 9, 609-612. [CrossRef] [PubMed]

3. Czaja, A.J. Frequency and nature of the variant syndromes of autoimmune liver disease. Hepatology 1998, 28, 360-365. [CrossRef] [PubMed]

4. Chazouillères, O.; Wendum, D.; Serfaty, L.; Montembault, S.; Rosmorduc, O.; Poupon, R. Primary biliary cirrhosis-autoimmune hepatitis overlap syndrome: Clinical features and response to therapy. Hepatology 1998, 28, 296-301. [CrossRef] [PubMed]

5. Lohse, A.W.; Büschenfelde, K.-H.M.Z.; Franz, B.; Kanzler, S.; Gerken, G.; Dienes, H.P. Characterization of the overlap syndrome of primary biliary cirrhosis $(\mathrm{PBC})$ and autoimmune hepatitis: Evidence for it being a hepatitic form of PBC in genetically susceptible individuals. Hepatology 1999, 29, 1078-1084. [CrossRef]

6. Chazouilleres, O.; Wendum, M.; Serfaty, L.; Rosmorduc, O.; Poupon, R. Long term outcome and response to therapy of primary biliary cirrhosis-Autoimmune hepatitis overlap syndrome. J. Hepatol. 2006, 44, 400-406. [CrossRef]

7. Poupon, R.; Chazouilleres, O.; Corpechot, C.; Chrétien, Y. Development of autoimmune hepatitis in patients with typical primary biliary cirrhosis. Hepatology 2006, 44, 85-90. [CrossRef]

8. Heurgué, A.; Vitry, F.; Diebold, M.-D.; Yaziji, N.; Bernard-Chabert, B.; Pennaforte, J.-L.; Picot, R.; Louvet, H.; Frémond, L.; Geoffroy, P.; et al. Overlap syndrome of primary biliary cirrhosis and autoimmune hepatitis: A retrospective study of 115 cases of autoimmune liver disease. Gastroentérologie Clin. Biol. 2007, 31, 17-25. [CrossRef]

9. Muratori, P.; Granito, A.; Pappas, G.; Pendino, G.M.; Quarneti, C.; Cicola, R.; Menichella, R.; Ferri, S.; Cassani, F.; Bianchi, F.B.; et al. The Serological Profile of the Autoimmune Hepatitis/Primary Biliary Cirrhosis Overlap Syndrome. Am. J. Gastroenterol. 2009, 104, 1420-1425. [CrossRef]

10. Ozaslan, E.; Efe, C.; Heurgué-Berlot, A.; Kav, T.; Masi, C.; Purnak, T.; Muratori, L.; Üstündă̆, Y.; Bresson-Hadni, S.; Thiefin, G.; et al. Factors Associated With Response to Therapy and Outcome of Patients With Primary Biliary Cirrhosis With Features of Autoimmune Hepatitis. Clin. Gastroenterol. Hepatol. 2014, 12, 863-869. [CrossRef]

11. Yang, F.; Wang, Q.; Wang, Z.; Miao, Q.; Xiao, X.; Tang, R.; Chen, X.; Bian, Z.; Zhang, H.; Yang, Y.; et al. The Natural History and Prognosis of Primary Biliary Cirrhosis with Clinical Features of Autoimmune Hepatitis. Clin. Rev. Allergy Immunol. 2015, 50, 114-123. [CrossRef]

12. Guo, C.; Zhang, H.; Yang, J.; Zhu, R.; Zheng, Y.; Dai, W.; Wang, F.; Chen, K.; Li, J.; Wang, C.; et al. Combination therapy of ursodeoxycholic acid and budesonide for PBC-AIH overlap syndrome: A meta-analysis. Drug Des. Dev. Ther. 2015, 9, 567-574. [CrossRef] [PubMed]

13. Joshi, S.; Cauch-Dudek, K.; Wanless, I.R.; Jorgensen, R.; Batts, K.; Heathcote, E.J.; Lindor, K.D. Primary biliary cirrhosis with additional features of autoimmune hepatitis: Response to therapy with ursodeoxycholic acid. Hepatology 2002, 35, 409-413. [CrossRef] [PubMed]

14. Ben-Ari, Z.; Dhillon, A.P.; Sherlock, S. Autoimmune cholangiopathy: Part of the spectrum of autoimmune chronic active hepatitis. Hepatology 1993, 18, 10-15. [CrossRef] [PubMed]

15. Wolf, D.C.; Bojito, L.; Facciuto, M.; Lebovics, E. Mycophenolate Mofetil for Autoimmune Hepatitis: A Single Practice Experience. Dig. Dis. Sci. 2008, 54, 2519-2522. [CrossRef] [PubMed]

16. Baven-Pronk, A.M.C.; Coenraad, M.J.; Van Buuren, H.R.; De Man, R.A.; Van Erpecum, K.J.; Lamers, M.M.H.; Drenth, J.P.H.; Berg, A.P.V.D.; Beuers, U.H.; Ouden, J.D.; et al. The role of mycophenolate mofetil in the management of autoimmune hepatitis and overlap syndromes. Aliment. Pharmacol. Ther. 2011, 34, 335-343. [CrossRef]

17. Duclos-Vallée, J.C.; Hadengue, A.; Ganne-Carrié, N.; Robin, E.; Degott, C.; Erlinger, S. Primary biliary cirrhosis-autoimmune hepatitis overlap syndrome. Corticoresistance and effective treatment by cyclosporine A. Dig. Dis. Sci. 1995, 40, 1069-1073. [CrossRef]

18. Neuhauser, M.; Bjornsson, E.; Treeprasertsuk, S.; Enders, F.; Silveira, M.; Talwalkar, J.; Lindor, K. Autoimmune Hepatitis-PBC Overlap Syndrome: A Simplified Scoring System May Assist in the Diagnosis. Am. J. Gastroenterol. 2010, 105, 345-353. [CrossRef] 
19. Martinez Casas, O.Y.; Diaz Ramirez, G.S.; Marin Zuluaga, J.I.; Santos, O.; Munoz Maya, O.; Donado Gomez, J.H.; Restrepo Gutierrez, J.C. Autoimmune hepatitis-Primary biliary cholangitis overlap syndrome. Long-term outcomes of a retrospective cohort in a university hospital. Gastroenterol. Hepatol. 2018, 41, 544-552. [CrossRef]

20. Silveira, M.G.; Talwalkar, J.A.; Angulo, P.; Lindor, K.D. Overlap of Autoimmune Hepatitis and Primary Biliary Cirrhosis: Long-Term Outcomes. Am. J. Gastroenterol. 2007, 102, 1244-1250. [CrossRef]

21. Kuiper, E.M.; Zondervan, P.E.; Van Buuren, H.R. Paris Criteria Are Effective in Diagnosis of Primary Biliary Cirrhosis and Autoimmune Hepatitis Overlap Syndrome. Clin. Gastroenterol. Hepatol. 2010, 8, 530-534. [CrossRef] [PubMed]

22. Lindgren, S.; Glaumann, H.; Almer, S.; Bergquist, A.; Björnsson, E.; Broomé, U.; Danielsson, Å.; Lebrun, B.; Prytz, H.; Olsson, R. Transitions between variant forms of primary biliary cirrhosis during long-term follow-up. Eur. J. Intern. Med. 2009, 20, 398-402. [CrossRef]

23. Campos, S.; Gomes, D.; Cipriano, M.A.; Sofia, C. The clinical extremes of autoimmune cholangitis. Rev. Española Enferm. Dig. 2017, 109, 465-468. [CrossRef] [PubMed]

24. Czaja, A.J.; Carpenter, H.A.; Santrach, P.J.; Moore, S.B. Autoimmune cholangitis within the spectrum of autoimmune liver disease. Hepatology 2000, 31, 1231-1238. [CrossRef]

25. Ozaslan, E.; Efe, C.; Akbulut, S.; Purnak, T.; Savas, B.; Erden, E.; Altiparmak, E. Therapy response and outcome of overlap syndromes: Autoimmune hepatitis and primary biliary cirrhosis compared to autoimmune hepatitis and autoimmune cholangitis. Hepatogastroenterology 2010, 57, 441-446. [PubMed]

26. Johnson, P.J.; McFarlane, I.G. Meeting report: International autoimmune hepatitis group. Hepatology 1993, 18, 998-1005. [CrossRef]

27. Alvarez, F.; Berg, P.; Bianchi, F.; Bianchi, L.; Burroughs, A.; Cançado, E.L.R.; Chapman, R.; Cooksley, W.; Czaja, A.; Desmet, V.; et al. International Autoimmune Hepatitis Group Report: Review of criteria for diagnosis of autoimmune hepatitis. J. Hepatol. 1999, 31, 929-938. [CrossRef]

28. Hennes, E.M.; Zeniya, M.; Czaja, A.J.; Parés, A.; Dalekos, G.N.; Krawitt, E.L.; Bittencourt, P.L.; Porta, G.; Boberg, K.M.; Hofer, H.; et al. Simplified criteria for the diagnosis of autoimmune hepatitis. Hepatology 2008, 48, 169-176. [CrossRef]

29. Chapman, R.; Fevery, J.; Kalloo, A.; Nagorney, D.M.; Boberg, K.M.; Shneider, B.; Gores, G.J. Diagnosis and management of primary sclerosing cholangitis. Hepatology 2009, 51, 660-678. [CrossRef]

30. Manns, M.P.; Czaja, A.J.; Gorham, J.D.; Krawitt, E.L.; Mieli-Vergani, G.; Vergani, D.; Ling, S.C. Diagnosis and management of autoimmune hepatitis. Hepatology 2010, 51, 2193-2213. [CrossRef]

31. EASL Clinical Practice Guidelines: Autoimmune hepatitis. J. Hepatol. 2015, 63, 971-1004. [CrossRef] [PubMed]

32. Gohlke, F.; Lohse, A.W.; Dienes, H.P.; Löhr, H.; Märker-Hermann, E.; Gerken, G.; Büschenfelde, K.-H.M.Z. Evidence for an overlap syndrome of autoimmune hepatitis and primary sclerosing cholangitis. J. Hepatol. 1996, 24, 699-705. [CrossRef]

33. Gregorio, G.; Portmann, B.; Karani, J.; Harrison, P.; Donaldson, P.T.; Vergani, D.; Mieli-Vergani, G. Autoimmune hepatitis/sclerosing cholangitis overlap syndrome in childhood: A 16-year prospective study. Hepatology 2001, 33, 544-553. [CrossRef] [PubMed]

34. Floreani, A.; Rizzotto, E.R.; Ferrara, F.; Carderi, I.; Caroli, D.; Blasone, L.; Baldo, V. Clinical Course and Outcome of Autoimmune Hepatitis/Primary Sclerosing Cholangitis Overlap Syndrome. Am. J. Gastroenterol. 2005, 100, 1516-1522. [CrossRef]

35. Lüth, S.; Kanzler, S.; Frenzel, C.; Kasper, H.U.; Dienes, H.P.; Schramm, C.; Galle, P.R.; Herkel, J.; Lohse, A.W. Characteristics and Long-term Prognosis of the Autoimmune Hepatitis/Primary Sclerosing Cholangitis Overlap Syndrome. J. Clin. Gastroenterol. 2009, 43, 75-80. [CrossRef]

36. Rodrigues, A.T.; Liu, P.M.F.; Fagundes, E.D.T.; Queiroz, T.C.N.; Barbosa, P.D.S.H.; Silva, S.L.C.; Silva, A.C.S.; Miranda, D.M.; Ferreira, A.R.; Alberti, L.R. Clinical Characteristics and Prognosis in Children and Adolescents With Autoimmune Hepatitis and Overlap Syndrome. J. Pediatr. Gastroenterol. Nutr. 2016, 63, 76-81. [CrossRef]

37. Igarashi, G.; Endo, T.; Mikami, K.; Sawada, N.; Satake, R.; Ohta, R.; Sakamoto, J.; Yoshimura, T.; Kurose, A.; Kijima, H.; et al. Two Cases of Primary Sclerosing Cholangitis Overlapping with Autoimmune Hepatitis in Adults. Intern. Med. 2017, 56, 509-515. [CrossRef] 
38. Lian, M.; Li, B.; Xiao, X.; Yang, Y.; Jiang, P.; Yan, L.; Sun, C.; Zhang, J.; Wei, Y.; Li, Y.; et al. Comparative clinical characteristics and natural history of three variants of sclerosing cholangitis: IgG4-related SC, PSC/AIH and PSC alone. Autoimmun. Rev. 2017, 16, 875-882. [CrossRef]

39. Abdo, A. Evolution of autoimmune hepatitis to primary sclerosing cholangitis: A sequential syndrome. Hepatology 2002, 36, 1393-1399. [CrossRef]

40. Olsson, R.; Glaumann, H.; Almer, S.; Broomé, U.; Lebrun, B.; Bergquist, A.; Björnsson, E.; Prytz, H.; Åke, D.; Lindgren, $\mathrm{S}$. High prevalence of small duct primary sclerosing cholangitis among patients with overlapping autoimmune hepatitis and primary sclerosing cholangitis. Eur. J. Intern. Med. 2009, 20, 190-196. [CrossRef]

41. McNair, A.N.; Moloney, M.; Portmann, B.C.; Williams, R.; McFarlane, I.G. Autoimmune hepatitis overlapping with primary sclerosing cholangitis in five cases. Am. J. Gastroenterol. 1998, 93, 777-784. [CrossRef] [PubMed]

42. van Buuren, H.R.; van Hoogstraten, H.J.E.; Terkivatan, T.; Schalm, S.W.; Vleggaar, F.P. High prevalence of autoimmune hepatitis among patients with primary sclerosing cholangitis. J. Hepatol. 2000, 33, 543-548. [CrossRef] [PubMed]

43. Ferrari, F.; Ranucci, G.; Aloi, M.; Della Volpe, L.; Viola, F.; Miele, E.; Cucchiara, S.; Iorio, R. A promising medium-term follow-up of pediatric sclerosing cholangitis: Mild phenotype or early diagnosis? Hepatol. Res. 2018, 48, 556-565. [CrossRef]

44. Smolka, V.; Karaskova, E.; Tkachyk, O.; Aiglova, K.; Ehrmann, J.; Michalkova, K.; Konecny, M.; Volejnikova, J. Long-term follow-up of children and adolescents with primary sclerosing cholangitis and autoimmune sclerosing cholangitis. Hepatobiliary Pancreat Dis. Int. 2016, 15, 412-418. [CrossRef]

45. Aw, M.M.; Dhawan, A.; Samyn, M.; Bargiota, A.; Mieli-Vergani, G. Mycophenolate mofetil as rescue treatment for autoimmune liver disease in children: A 5-year follow-up. J. Hepatol. 2009, 51, 156-160. [CrossRef]

46. Al-Chalabi, T.; Portmann, B.C.; Bernal, W.; McFarlane, I.G.; Heneghan, M.A. Autoimmune hepatitis overlap syndromes: An evaluation of treatment response, long-term outcome and survival. Aliment. Pharm. Ther. 2008, 28, 209-220. [CrossRef]

47. Burak, K.; Urbanski, S.J.; Swain, M.G. CASE REPORT: A Case of Coexisting Primary Biliary Cirrhosis and Primary Sclerosing Cholangitis: A New Overlap of Autoimmune Liver Diseases. Dig. Dis. Sci. 2001, 46, 2043-2047. [CrossRef]

48. Floreani, A.; Motta, R.; Cazzagon, N.; Franceschet, I.; Roncalli, M.; Del Ross, T.; Rosina, F.; Lleo, A.; Mescoli, C.; Colloredo, G.; et al. The overlap syndrome between primary biliary cirrhosis and primary sclerosing cholangitis. Dig. Liver Dis. 2015, 47, 432-435. [CrossRef]

49. Jeevagan, A. Overlap of primary biliary cirrhosis and primary sclerosing cholangitis-A rare coincidence or a new syndrome. Int. J. Gen. Med. 2010, 3, 143-146. [CrossRef]

50. Kingham, J.G.; Abbasi, A. Co-existence of primary biliary cirrhosis and primary sclerosing cholangitis: A rare overlap syndrome put in perspective. Eur. J. Gastroenterol. Hepatol. 2005, 17, 1077-1080. [CrossRef]

51. Mandolesi, D.; Lenzi, M.; D’Errico, A.; Festi, D.; Bazzoli, F.; Colecchia, A. Primary biliary cholangitis-primary sclerosing cholangitis in an evolving overlap syndrome: A case report. Gastroenterol. Hepatol. 2017, 40, 669-671. [CrossRef] [PubMed]

52. Oliveira, E.M.G.; Oliveira, P.M.; Becker, V.; Dellavance, A.; Andrade, L.E.C.; Lanzoni, V.; Silva, A.E.B.; Ferraz, M.L.G. Overlapping of Primary Biliary Cirrhosis and Small Duct Primary Sclerosing Cholangitis: First Case Report. J. Clin. Med. Res. 2012, 4, 429-433. [CrossRef] [PubMed]

53. Sundaram, S.; Shankar, K.; Mazumdar, S.; Shukla, A. Overlap Syndrome between Primary Biliary Cholangitis and Primary Sclerosing Cholangitis. ACG Case Rep. J. 2018, 5, e54. [CrossRef]

54. Alghamdi, W.; Qumosani, K. A195 triple overlap syndrome? A rare case of aih, pbc and psc overlap. J. Can. Assoc. Gastroenterol. 2018, 1, 287. [CrossRef]

55. Gao, S.J.; Xu, Y.; Bao, J.; Liu, X.; Liu, X.M.; Hua, J. Diagnosis and Treatment of Trigeminy Overlap Syndrome of Autoimmune Liver Disease. World Chin. J. Dig. 2016, 24, 293-298.

56. Zhang, Y.; Lu, J.; Dai, W.; Wang, F.; Shen, M.; Yang, J.; Zhu, R.; Zhang, H.; Chen, K.; Cheng, P.; et al. Combination Therapy of Ursodeoxycholic Acid and Corticosteroids for Primary Biliary Cirrhosis with Features of Autoimmune Hepatitis: A Meta-Analysis. Gastroenterol. Res. Pract. 2013, 2013, 1-9. [CrossRef] [PubMed]

57. Günsar, F.; Akarca, U.S.; Ersöz, G.; Karasu, Z.; Yüce, G.; Batur, Y. Clinical and biochemical features and therapy responses in primary biliary cirrhosis and primary biliary cirrhosis-autoimmune hepatitis overlap syndrome. Hepatogastroenterology 2002, 49, 1195-1200. 
58. Tanaka, A.; Harada, K.; Ebinuma, H.; Komori, A.; Yokokawa, J.; Yoshizawa, K.; Abe, M.; Miyake, Y.; Kikuchi, K.; Ohira, H.; et al. Primary biliary cirrhosis-Autoimmune hepatitis overlap syndrome: A rationale for corticosteroids use based on a nation-wide retrospective study in Japan. Hepatol. Res. 2011, 41. [CrossRef]

59. Hirschfield, G.; Beuers, U.; Corpechot, C.; Invernizzi, P.; Jones, D.; Marzioni, M.; Schramm, C. EASL Clinical Practice Guidelines: The diagnosis and management of patients with primary biliary cholangitis. J. Hepatol. 2017, 67, 145-172. [CrossRef]

60. Lindor, K.D.; Bowlus, C.L.; Boyer, J.; Levy, C.; Mayo, M. Primary Biliary Cholangitis: 2018 Practice Guidance from the American Association for the Study of Liver Diseases. Clin. Liver Dis. 2020, 15, 1-2. [CrossRef]

61. Moher, D.; Liberati, A.; Tetzlaff, J.; Altman, D.G. Preferred reporting items for systematic reviews and meta-analyses: The PRISMA statement. PLoS Med. 2009, 6, e1000097. [CrossRef] [PubMed]

62. Wells, G.A.; Shea, B.; O'Connell, D.; Peterson, J.; Welch, V.; Losos, M.; Tugwell, P. The Newcastle-Ottawa Scale (Nos) for Assessing the Quality of Nonrandomised Studies in Metaanalyses. Available online: http://www.ohri.ca/programs/clinical_epidemiology/oxford.asp (accessed on 1 November 2019).

63. Schünemann, H.; Brożek, J.; Guyatt, G.; Oxman, A. GRADE Handbook. Available online: https://gdt. gradepro.org/app/handbook/handbook.html (accessed on 24 March 2020).

64. Cheikh, I.; Said, Y.; Chaabouni, H.; Ouerghi, H.; Ben Ammar, A. Primary biliary cirrhosis and autoimmune hepatitis overlap syndrome: Therapeutic features in 5 patients. Ann. Med. Interne 2003, 154, 7-11.

65. Efe, C.; Ozaslan, E.; Heurgue-Berlot, A.; Kav, T.; Masi, C.; Purnak, T.; Torgutalp, M.; Muratori, L.; Bresson-Hadni, S.; Thiefin, G.; et al. Sequential presentation of primary biliary cirrhosis and autoimmune hepatitis. Eur. J. Gastroenterol. Hepatol. 2014, 26, 532-537. [CrossRef] [PubMed]

66. Fan, X.; Zhu, Y.; Men, R.; Wen, M.; Shen, Y.; Lu, C.; Yang, L. Efficacy and Safety of Immunosuppressive Therapy for PBC-AIH Overlap Syndrome Accompanied by Decompensated Cirrhosis: A Real-World Study. Can. J. Gastroenterol. Hepatol. 2018, 2018, 1-9. [CrossRef] [PubMed]

67. Levy, C.; Naik, J.; Giordano, C.; Mandalia, A.; O’Brien, C.; Bhamidimarri, K.R.; Schiff, E.R.; Martin, P. Hispanics With Primary Biliary Cirrhosis Are More Likely to Have Features of Autoimmune Hepatitis and Reduced Response to Ursodeoxycholic Acid Than Non-Hispanics. Clin. Gastroenterol. Hepatol. 2014, 12, 1398-1405. [CrossRef] [PubMed]

68. Liu, C.; Xu, C.; Li, G.; Chi, B. Analysis of clinical features of patients with autoimmune hepatitis/primary biliary cirrhosis overlap syndrome. J. Jilin Univ. Med. 2014, 40, 646-649.

69. Liu, F.; Pan, Z.G.; Ye, J.; Xu, N.; Guo, H.; Li, G.P.; Xu, K.S.; Hou, X.H.; Song, Y. Primary biliary cirrhosis-autoimmune hepatitis overlap syndrome: Simplified criteria may be effective in the diagnosis in Chinese patients. J. Dig. Dis. 2014, 15, 660-668. [CrossRef]

70. Park, Y.; Cho, Y.; Cho, E.J.; Kim, Y.J. Retrospective analysis of autoimmune hepatitis-primary biliary cirrhosis overlap syndrome in Korea: Characteristics, treatments, and outcomes. Clin. Mol. Hepatol. 2015, 21, 150-157. [CrossRef]

71. Saito, H.; Rai, T.; Takahashi, A.; Kanno, Y.; Monoe, K.; Irisawa, A.; Ohira, H. Clinicolaboratory Characteristics of Japanese Patients with Primary Biliary Cirrhosis-Autoimmune Hepatitis Overlap. Fukushima J. Med Sci. 2006, 52, 71-77. [CrossRef]

72. Serghini, M.; Haddad, W.; Karoui, S.; Ben Mustapha, N.; Kallel, L.; Fekih, M.; Boubaker, J.; Filali, A. Overlap syndrome of primary biliary cirrhosis and autoimmune hepatitis. La Tunis. Medicale 2012, 90, 741-743.

73. Wu, C.-H.; Wang, Q.-H.; Tian, G.-S.; Xu, X.-Y.; Yu, Y.-Y.; Wang, G.-Q. Clinical features of the overlap syndrome of autoimmune hepatitis and primary biliary cirrhosis: Retrospective study. Chin. Med. J. 2006, 119, $238-241$. [CrossRef] [PubMed]

74. Yokokawa, J.; Saito, H.; Kanno, Y.; Honma, F.; Monoe, K.; Sakamoto, N.; Abe, K.; Takahashi, A.; Yokokawa, H.; Ohira, H. Overlap of primary biliary cirrhosis and autoimmune hepatitis: Characteristics, therapy, and long term outcomes. J. Gastroenterol. Hepatol. 2010, 25, 376-382. [CrossRef] [PubMed]

75. Yoshioka, Y.; Taniai, M.; Hashimoto, E.; Haruta, I.; Shiratori, K. Clinical profile of primary biliary cirrhosis with features of autoimmune hepatitis: Importance of corticosteroid therapy. Hepatol. Res. 2013, 44, 947-955. [CrossRef] [PubMed]

76. Efe, C.; Ozaslan, E.; Kav, T.; Purnak, T.; Shorbagi, A.; Ozkayar, O.; Berlot, A.H.; Sokmensuer, C.; Muratori, P. Liver fibrosis may reduce the efficacy of budesonide in the treatment of autoimmune hepatitis and overlap syndrome. Autoimmun. Rev. 2012, 11, 330-334. [CrossRef]

77. Heathcote, E.J. Management of primary biliary cirrhosis. Hepatology 2000, 31, 1005-1013. [CrossRef] 
78. Ludwig, J.; Czaja, A.J.; Dickson, E.R.; LaRusso, N.F.; Wiesner, R.H. Manifestations of nonsuppurative cholangitis in chronic hepatobiliary diseases: Morphologic spectrum, clinical correlations and terminology. Liver 1984, 4, 105-116. [CrossRef]

79. Batts, K.P.; Ludwig, J. An Update on Terminology and Reporting. Am. J. Surg. Pathol. 1995, 19, $1409-1417$. [CrossRef]

80. Czaja, A.J. Diagnosis, prognosis, and treatment of classical autoimmune chronic active hepatitis. In Autoimmune Liver Disease; Raven: New York, NY, USA, 1991; pp. 143-166.

81. Ishak, K.; Baptista, A.; Bianchi, L.; Callea, F.; De Groote, J.; Gudat, F.; Denk, H.; Desmet, V.; Korb, G.; Macsween, R.N.; et al. Histological grading and staging of chronic hepatitis. J. Hepatol. 1995, 22, 696-699. [CrossRef]

82. Mileti, E.; Rosenthal, P.; Peters, M.G. Validation and Modification of Simplified Diagnostic Criteria for Autoimmune Hepatitis in Children. Clin. Gastroenterol. Hepatol. 2011, 10, 417-421.e1. [CrossRef]

83. Bedossa, P.; Poynard, T. An algorithm for the grading of activity in chronic hepatitis C. The METAVIR Cooperative Study Group. Hepatology 1996, 24, 289-293. [CrossRef]

84. Lee, J.Y.; Danford, C.J.; Trivedi, H.D.; Tapper, E.B.; Patwardhan, V.R.; Bonder, A. Treatment of Fatigue in Primary Biliary Cholangitis: A Systematic Review and Meta-Analysis. Dig. Dis. Sci. 2019, 64, 2338-2350. [CrossRef] [PubMed]

(C) 2020 by the authors. Licensee MDPI, Basel, Switzerland. This article is an open access article distributed under the terms and conditions of the Creative Commons Attribution (CC BY) license (http://creativecommons.org/licenses/by/4.0/). 